\title{
La adaptación del Sistema Nacional de Incentivos Regionales a la Ley para el Desarrollo Sostenible del Medio Rural
}

\author{
PEDRO BENITO MOYANO PESQUERA \\ Departamento de Economía Aplicada. UNIVERSIDAD DE VALLADOLID, ESPAÑA. E- \\ mail: moyano@eco.uva.es
}

\section{RESUMEN}

En enero de 2008 entró en vigor la Ley para el desarrollo sostenible del medio rural. Con el objetivo de fomentar la actividad económica en estas áreas, propone que el sistema nacional de incentivos regionales dé un trato preferente a las zonas rurales consideradas prioritarias. En junio de 2010 se aprueba el Programa de Desarrollo Rural Sostenible para el periodo 2010-2014 y con él, los incentivos regionales adquieren el papel protagonista como instrumento al servicio del desarrollo rural.

Este artículo tiene por finalidad contrastar si los incentivos regionales concedidos entre 2008 y 2010 muestran algún tipo de discriminación positiva a favor de las zonas rurales, en general, y de las menos desarrolladas en particular. Es decir, si han comenzado a adaptarse al nuevo escenario en el que deberán representar su papel de manera efectiva desde 2010.

Palabras clave: Desarrollo rural, incentivos regionales, inversión, empleo, delimitación territorial.

\section{Adjustment of the National System of Regional Incentives to the Law for Sustainable Development in Rural Environment}

\begin{abstract}
In January 2008, the Law for sustainable development in rural environment came into force. Aimed at promoting economic activity in rural areas, the Law proposes that the National System of Regional Incentives should give preferential treatment to rural areas considered to be priority. In June 2010, the 2010-2014 Sustainable Rural Development Programme was approved, through which regional incentives take on a leading role as an instrument to serve rural development.

The objective of this paper is to ascertain whether the regional incentives granted between 2008 and 2010 evidence any positive discrimination in favour of rural zones in general, and particularly least developed areas, in other words whether they have begun to adapt to the new scene in which they will have to play their role effectively as of 2010.
\end{abstract}

Keywords: Rural development, regional incentives, employment, investment

Clasificación JEL: E62, L52, R11, R58

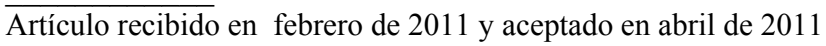

Artículo disponible en versión electrónica en la página www.revista-eea.net, ref. ə-29108 


\section{INTRODUCCIÓN}

El 3 de enero de 2008 entró en vigor la Ley 45/2007 de 13 de diciembre para el desarrollo sostenible del medio rural (BOE ${ }^{\circ} 299$, de 14 de diciembre) entre cuyos objetivos generales (art.2.1.a) figura " ${ }^{1}$ "Mantener y ampliar la base económica del medio rural mediante la preservación de actividades competitivas y multifuncionales, y la diversificación de su economía con la incorporación de nuevas actividades compatibles con un desarrollo sostenible". Además, señala expresamente que las políticas de desarrollo rural sostenible derivadas de esta Ley deberán orientarse, entre otros objetivos particulares, a (art.2.2.a) "Fomentar una actividad económica continuada y diversificada en el medio rural, manteniendo un sector agrícola, ganadero, forestal y derivado de la pesca e impulsando la creación y el mantenimiento del empleo y renta en otros sectores, preferentemente en las zonas rurales consideradas prioritarias".

Para fomentar la actividad económica en el medio rural, la Ley propone que el Sistema Nacional de Incentivos Regionales trate de manera preferente a los proyectos que se desarrollen en las zonas rurales consideradas prioritarias y que éstas reciban una atención diferenciada en los planes nacionales de fomento empresarial. La primera opción engarza con la permanente preocupación que la Unión Europea mantiene desde sus orígenes por cualquier amenaza que falsee la competencia empresarial. La segunda conduce a la recurrente pregunta de por qué un individuo decide asumir los riesgos que conlleva la aventura de crear una empresa. Los economistas han tratado, a lo largo de la historia, de contestar esta cuestión desde un amplio abanico de enfoques. Riesgo asociado a: la incertidumbre (Cantillon, Von Thünen, Mill, Hawley, Knight, Von Mises, Cole, Shackle), ofrecer capital financiero (Smith, Bóhm-Bawerk, Pigou, Von Mises), innovar (Bentham, Von Thünen, Schmoller, Sombart, Weber, Schumpeter, Shakle), tomar decisiones (Say, Saint-Simon, Von Mises, Cole, Schultz), liderar (Say, Saint-Simon, Marshall, Sombart, Weber, Schumpeter), coordinar y gestionar recursos económicos (Say, Sombart, Weber, Coase, Schumpeter), emplear factores de producción (Walter, Wieser, Keynes), asignar recursos entre usos alternativos (Cantillon, Schultz).

El fomento de la actividad económica, especialmente en el medio rural, no puede abordarse sin considerar los factores determinantes en la creación de

1 Además, también se persigue "Mantener y mejorar el nivel de población del medio rural y elevar el grado de bienestar de sus ciudadanos, asegurando unos servicios públicos básicos adecuados y suficientes que garanticen la igualdad de oportunidades y la no discriminación, especialmente de las personas más vulnerables o en riesgo de exclusión" (art. 2.1.b) y "Conservar y recuperar el patrimonio y los recursos naturales y culturales del medio rural a través de actuaciones públicas y privadas que permitan su utilización compatible con un desarrollo sostenible" (art. 2.1.c). 
nuevas empresas. Muchos han sido los estudios que lo han intentado ${ }^{2}$, cada uno de ellos con distintos enfoques metodológicos, sectoriales, territoriales y temporales. Pese a la disparidad de las variables que, finalmente se consideran relevantes en la natalidad empresarial (Aleixandre y Moyano, 2009 y 2010), en la mayoría de ellos emerge un conjunto de factores comunes relativos a la población (volumen, características y distribución en el territorio), estructura empresarial (tamaño empresarial y densidad industrial) y mercado laboral (cualificación y tasas de paro). Los estudios muestran que el primer elemento indispensable en el proceso creador de nuevas empresas es la existencia de población, de cuyo seno surja alguien que quiera emprender (es decir, asumir riesgos). Sin población, no hay empresarios y sin éstos no hay empresas. No obstante, la necesidad de que exista una determinada "masa crítica", sea de población, sea de número de empresas ya existentes, es una condición necesaria, aunque no suficiente para estimular la deseada natalidad empresarial. La ausencia de esta condición previa, en general, no se suple con incentivos. Son numerosos los estudios que señalan que éstos no representan un factor determinante en las decisiones de localización empresarial porque no contrarrestan las desventajas derivadas de determinadas políticas macroeconómicas o de ausencia de otros factores.

La utilización de los incentivos regionales con el objetivo de fomentar la actividad económica en el medio rural no comienza con la aprobación de la Ley para el Desarrollo Sostenible del Medio Rural. De hecho, no es sino hasta el 5 de junio de $2010^{3}$, con la entrada en vigor del primer Programa de Desarrollo Rural Sostenible para el periodo 2010-2014, cuando los incentivos regionales adoptan un papel protagonista como instrumento al servicio del desarrollo rural. El Programa de Desarrollo Rural Sostenible plurianual constituye el principal instrumento de aplicación de la Ley 45/2007, tal y como se indica en el preámbulo de ésta. Teniendo en cuenta el poco tiempo transcurrido y el escaso

2 Entre los ejemplos más representativos podemos citar los trabajos que intentan aproximarse a la natalidad empresarial a través de nuevas empresas por cada 1.000 personas entre 15 y 64 años de edad (Davidsson, Lindmark y Olofsson, 1994), nuevas empresas por cada 1.000 residentes (Garafoli, 1994), tasa de autoempleo (Georgellis y Wall, 2002), nuevas empresas por número trabajadores (Kangasharju, 2000; Armigton y Acs, 2002), nuevas empresas por número de empresas existentes (Audretsch y Fritsch, 1994 y 1999; Guesnier, 1994; Keeble y Walker, 1994; Nyström, 2007), nuevas empresas por distrito (Bade y Nerlinger, 2000), nuevas empresas por cada 1.000 activos (Guesnier, 1994; Hart y Gudgin, 1994; Keeble y Walker, 1994; Fritsch y Mueller, 2007).

3 Real Decreto 752/2010, de 4 de junio, por el que se aprueba el primer Programa de Desarrollo Rural Sostenible para el período 2010-2014 en aplicación de la Ley 45/2007, de 13 de diciembre, para el desarrollo sostenible del medio rural. (BOE $\mathrm{n}^{\circ} 142$, de 11 de junio de 2010). 
número de incentivos concedidos ${ }^{4}$ no es viable ni operativo realizar un análisis de la eficacia y de la eficiencia de su utilización como instrumento para el desarrollo rural ${ }^{5}$. Además, esta evaluación sólo podrá afrontarse una vez que pase el tiempo suficiente para comprobar si los incentivos concedidos se han pagado total o parcialmente, pues la experiencia acumulada desde el origen ${ }^{6}$ de la Ley 50/1985 demuestra que existen múltiples causas por las que la ayuda inicial se minora o, incluso, se anula íntegramente. Es muy elevado el porcentaje de proyectos aprobados que, con el paso del tiempo, caducan, decaen, incumplen alguno de los términos o son modificados por la propia administración (Moyano, Aleixandre y Ogando, 2006; Moyano y Ogando, 2003).

Por ello, en este artículo se pretende contrastar si los incentivos regionales concedidos desde la aprobación de la Ley 45/2007 hasta su aplicación efectiva como instrumento de desarrollo rural, es decir entre 2008 y 2010 , muestran indicios que permitan aventurar algún tipo de discriminación positiva hacia las zonas rurales, particularmente hacia aquellas a revitalizar. En definitiva, si en estos tres años, los incentivos regionales han comenzado a adaptarse al nuevo escenario. Previamente al análisis, se detallan, en primer lugar, las principales características de la Ley de Incentivos Regionales, pues no hay que olvidar que en el diseño inicial de ésta no estaba presente como objetivo el desarrollo rural y, en segundo término, se establece el ámbito territorial de aplicación de la Ley de Desarrollo Sostenible del Medio Rural para comprobar la adecuación de ésta al ámbito territorial de aplicación de aquella.

Para contrastar si existen diferencias estadísticamente significativas en las características tanto de los proyectos incentivados como de las subvenciones concedidas, entre los cuatro tipos de zonas consideradas (a revitalizar, intermedias, periurbanas y urbanas), se utilizará el procedimiento de

4 Desde su entrada en vigor y hasta diciembre de 2010 sólo se han incentivado 78 proyectos de inversión, 70 mediante la Orden EHA/2132/2010, de 30 de junio, (BOE ${ }^{\circ} 188$ de agosto de 2010) y 8 a través de la Orden EHA/2733/2010, de 8 de octubre, (BOE n 257 de 23 de octubre de 2010).

5 El Programa de Desarrollo Rural Sostenible establece para el seguimiento y evaluación de la medida de fomento de la actividad económica en el medio rural a través de incentivos económicos regionales tres indicadores de ejecución y seguimiento: número de zonas rurales con actuación; número de proyectos pagados de incentivos regionales localizados en las zonas rurales prioritarias dentro de las zonas de promoción económica de incentivos regionales y subvención pagada a proyectos localizados en las zonas rurales prioritarias dentro de las zonas de promoción económica de incentivos regionales

6 La primera concesión de incentivos se realiza mediante Resolución de 7 de octubre de 1988 de la Dirección General de Incentivos Económicos Regionales por la que se hace pública la Orden del Ministerio de Economía y Hacienda por la que se resuelven solicitudes de beneficios en las Zonas de Promoción Económica, mediante la resolución de cuatro expedientes (BOE $\mathrm{n}^{\circ} 256$ de 25 de octubre de 1988). 
comparación de medias (Anova y prueba T). Anova mide la significación de la influencia de una o varias variables independientes no métricas sobre otra variable dependiente y métrica, contrastando diferencias entre las medias de varias muestras o grupos. Por tanto, permite determinar si diversos conjuntos de muestras aleatorias de una variable proceden de la misma población o no. En este sentido, es una extensión de la prueba $\mathrm{T}$ para dos muestras, que compara las medias de dos grupos de casos. Además de determinar la significación de la influencia de las modalidades de la/s variable/s no métrica sobre la variable métrica, en el caso de que ésta exista, Anova permite descubrir qué modalidades provocan los cambios.

Este análisis ha precisado recoger directamente del Boletín Oficial del Estado los datos de las Órdenes de Resolución de las solicitudes de proyectos acogidos a la Ley 50/1985 comprendidas entre enero de 2000 y diciembre de 2010. En total se analizan los 5.720 incentivos concedidos en las 57 Órdenes publicadas entre la Orden de 25 de febrero de 2000 (BOE n ${ }^{\circ} 72$ de 24 de marzo) y la Orden EHA/2733/2010 (BOE n 257 de 23 de octubre). En concreto, para el periodo comprendido entre 2000 y 2002 el número de incentivos concedidos fue de 1.917, mientras que para el trienio 2008-2010 los incentivos fueron 627.

\section{EL SISTEMA NACIONAL DE INCENTIVOS REGIONALES: LA LEY 50/1985}

La política de incentivos regionales en España se inicia con la Ley 50/1985, de 27 de diciembre, de incentivos regionales para la corrección de los desequilibrios económicos territoriales, desarrollada por el Reglamento de ejecución aprobado por Real Decreto 1535/1987 de 11 de diciembre, donde se contemplan las líneas generales del sistema de acuerdo con la normativa comunitaria (Comisión Europea, 1979). Se consideran incentivos regionales "las ayudas financieras que conceda el Estado para fomentar la actividad empresarial y orientar su localización hacia zonas previamente determinadas al objeto de reducir las diferencias de situación económica en el territorio nacional, repartir más equilibradamente las actividades económicas sobre el mismo y reforzar el potencial de desarrollo endógeno de las regiones". Los incentivos contemplados son de dos tipos: subvenciones y bonificaciones en la cuota empresarial a la Seguridad Social, si bien, en el desarrollo de la Ley, se opta por la primera de las modalidades. Es decir, a pesar del amplio abanico de modalidades de ayudas regionales que contempla la Comunidad Europea, la Ley 50/1985 se decanta, exclusivamente, por las subvenciones a empresas a fondo perdido.

Inicialmente, para alcanzar el equilibrio económico territorial, se establecen tres tipos de zonas: Zonas de Promoción Económica, en los territorios con un menor nivel de desarrollo; Zonas Industrializadas en Declive, en territorios 
afectados por importantes procesos de ajuste industrial, con graves repercusiones sobre el nivel de actividad y de empleo en la industria de la correspondiente zona; y, Zonas Especiales, que pueden ser creadas por el Gobierno cuando existan circunstancias que así lo justifiquen. A su vez, dependiendo del nivel de desarrollo, el territorio se divide en zonas tipo I, tipo II, tipo III y tipo IV, siendo los topes máximos de subvención a la inversión aprobada del $50 \%, 40 \%, 30 \%$ y $20 \%$, respectivamente. Únicamente en las zonas tipo I, II y III pueden crearse Zonas de Promoción Económica y sólo dentro de éstas se determinan zonas prioritarias en función de su población, accesibilidad, disponibilidad de suelo industrial y dotación de equipamiento. Los proyectos de inversión localizados en las zonas prioritarias tienen la posibilidad de recibir el importe máximo de la subvención ${ }^{7}$.

El diseño inicial ${ }^{8}$ abarcó un total de 6.030 municipios, el 95,6\% de ellos ubicados en Zonas de Promoción Económica repartidos en trece Comunidades Autónomas, todas ellas incentivables en el $100 \%$ de su territorio, salvo Aragón, Comunidad Valenciana y País Vasco. En total, el 96,4\% del territorio español podía, en mayor o menor grado, acogerse a incentivos regionales. Esta situación no fue el punto final en la delimitación territorial de las áreas, sino el comienzo de sucesivas modificaciones que han demarcado, definido, modificado y prorrogado, las diferentes zonas, figuras y porcentajes de subvención con el fin de adaptarlas a los cambios en las Directrices sobre ayudas de Estado de finalidad regional para el periodo 2000-2006 (Comisión Europea, 1995, 1998, 2000) y en las Directrices para el periodo 2007-2013 (Comisión Europea, 2006, 2007).

En 2007 se aprueba un nuevo Reglamento de Desarrollo de la Ley de Incentivos Regionales ${ }^{9}$ que revisa, actualiza y unifica la normativa de los incentivos regionales. El nuevo Reglamento, unido a las anteriores modificaciones de los Reales Decretos de Delimitación de Zona, hace necesario acotar, de nuevo, las zonas incentivables. Con este motivo, en marzo de 2008 se publican los trece Reales Decretos que delimitan las zonas de promoción económica incentivables: Andalucía, Aragón, Asturias, Canarias, Cantabria, Castilla-La Mancha, Castilla y León, Ceuta, Comunidad Valenciana, Extremadura, Galicia, Melilla y Murcia. Estas son, por tanto, las once

7 Las Zonas Industrializadas en Declive y las Zonas Especiales también podían recibir porcentajes superiores a los indicados. Incluso, con carácter excepcional, podían autorizarse elevaciones en los topes máximos de las zonas tipo I y II.

8 Con la entrada en vigor del Real Decreto 21/1988, de 21 de enero, de Delimitación de la Zona Industrializada en Declive de Ferrol comienza a perfilarse el diseño de las zonas incentivables de España que culmina con el Real Decreto 883/1989, de 14 de julio, de Delimitación de la Zona de Promoción Económica de la Comunidad Valenciana.

9 Real Decreto 899/2007, de 6 de julio (BOE no 172 de 19 de julio de 2007). 
Comunidades y dos Ciudades Autónomas sobre las que poder aplicar el instrumento de incentivos regionales propugnado por la Ley 45/2007 para el Desarrollo Sostenible del Medio Rural.

La existencia de diferentes zonas en función de circunstancias y problemas dispares se refleja en el diferente porcentaje máximo de subvención a la inversión aprobada al que se puede acceder. Este porcentaje oscila entre el $40 \%$ en Andalucía, Canarias, Extremadura y Galicia, y el 15\% en Cantabria y parte de Aragón. Paralelamente, se establecen zonas prioritarias en las que el porcentaje de subvención que correspondería al proyecto por la aplicación de los criterios, se incrementa un $20 \%$, respetando siempre el límite máximo determinado.

A pesar de estas diferencias, todos los Reales Decretos de delimitación de las trece zonas, coinciden en objetivos, sectores y elementos incentivables. El objetivo común en todas ellas es el de impulsar y desarrollar el tejido socioeconómico con especial atención al aumento del nivel de vida en el territorio, en particular en sus zonas más deprimidas ${ }^{10}$. Los sectores productivos sobre los que se trata de incidir comprenden las industrias transformadoras y servicios de apoyo a la producción y los establecimientos turísticos e instalaciones complementarias de ocio ${ }^{11}$, siendo inversiones incentivables las realizadas en obra civil, bienes de equipo y estudios previos de los proyectos de creación de nuevos establecimientos, ampliación de una actividad ya establecida o modernización de las instalaciones.

\section{LA IMPORTANCIA (Y DIFICULTAD) DE LA DELIMITACIÓN TERRITORIAL}

La controversia en torno a lo que se entiende por espacio rural y espacio urbano no ha cesado desde mediados del siglo pasado. Tal es así que, en no

${ }^{10}$ A través de "a) La promoción en la creación de empresas innovadoras y de base tecnológica que propongan inversiones basadas en proyectos de investigación, desarrollo e innovación (I+ $D+I) y$, en general, en la innovación tecnológica, el diseño industrial y la mejora medioambiental. b) El impulso del potencial endógeno del territorio así como desarrollar y consolidar el tejido industrial en base a criterios de calidad, eficiencia, productividad y respeto al medio ambiente. c) El fomento de la diversificación en los sectores de la producción y la distribución que aumenten el atractivo y el impulso de la actividad en el territorio".

11 Además de esta demarcación, en todos los casos se faculta a los órganos competentes para que, excepcionalmente, y previo informe del Consejo Rector puedan conceder incentivos regionales a proyectos que, no estando incluidos en los sectores mencionados, contribuyan de una forma significativa al logro de los objetivos especificados. El Consejo Rector, órgano adscrito al Ministerio de Economía y Hacienda, está encargado de programar y promover las actuaciones estatales en materia de incentivos regionales, de velar por la coordinación de esos incentivos con los restantes instrumentos de desarrollo regional y con las ayudas sectoriales con incidencia regional. 
pocas ocasiones, el término rural se define de una forma ad hoc, en función de aquello que se pretende estudiar. Aunque este debate parezca, con frecuencia baldío, el diseño y la puesta en práctica de determinadas políticas públicas hace imprescindible distinguir lo rural de lo urbano. Elegir el criterio por el que se delimitan las zonas rurales no es un mero divertimento teórico. Ser o no ser rural significa en muchos casos que los municipios tengan o no tengan acceso directo o indirecto a determinados servicios, subvenciones o ayudas.

La delimitación del medio rural puede realizarse a través de un criterio específico para estas áreas o llegar a ellas por exclusión; es decir, acotar las zonas rurales después de determinar las áreas urbanas, comprendiendo aquéllas todo el territorio no urbano. Aunque la zona urbana ha sido considerada sinónimo de ciudad, establecer dónde comienza y dónde acaba es un asunto cada vez más arduo de precisar. En general, la dicotomía territorial olvida la relación que se establece entre municipios y, en particular, el hecho frecuente, en la mayoría de las ciudades, de no considerar parte del área urbana los pequeños municipios adyacentes a dichas localidades. La realidad muestra la existencia de áreas formadas por varias entidades de población que, aunque independientes desde el punto de vista administrativo, presentan indudables vínculos económicos, además de formar espacios de vida y trabajo comunes de los ciudadanos. Como recuerda Serrano (2006), frente a la ciudad, de núcleo compacto, cerrado, amurallada en la época medieval, y hasta hace poco rodeada y delimitada por sus barrios, cada vez se trata más de una entidad morfológica diferente, donde sus periferias se difuminan con perfiles menos nítidos. Limitar tales cuestiones a sus dimensiones administrativas, formales, suele ser una forma de actuación frecuente, por sencilla, pero no exenta de riesgos e imprecisiones.

Los procesos urbanos adquieren una dimensión y escala que superan las estructuras y formas de ciudad tradicional y los límites administrativos municipales, para englobar, mediante diferentes formas de articulación espacial, asentamientos de población y territorios cada vez más amplios (Feria, 2004). Aceptar una visión dicotómica (rural-urbana) de la realidad significa para Rodríguez y Saborío (2008): limitar el conocimiento de las poblaciones con características intermedias, introduciendo ambigüedad en la clasificación de este tipo de territorios; impedir un adecuado conocimiento de la dinámica de los territorios, especialmente de su vinculación económica y de las características de su desarrollo; no permitir ver las interdependencias entre territorios, favoreciendo enfoques urbano-centristas (por ejemplo, sesgo urbano en el gasto público); no facilitar el análisis de los flujos comerciales; no permitir ver cambios espaciales en los patrones de empleo; limitar las posibilidades de analizar la relación entre cambios en el patrón de asentamientos humanos y las demandas de servicios públicos. 
Tampoco se puede apelar, como tradicionalmente se ha hecho, a las funciones terciarias que presta la ciudad como elemento de la esencia urbana. En la actualidad, las actividades terciarias están presentes incluso en pequeños asentamientos de población de dudosa naturaleza urbana. Como recuerda Tió (2005), históricamente la caracterización de las zonas rurales se ha basado en el predominio de las actividades agrarias, asociadas a modos de vida y de relaciones humanas y familiares más permanentes, rígidos y anclados en modelos sociales tradicionales. Sin embargo, el desarrollo de los sistemas de infraestructuras, comunicaciones y transmisión de la información ha supuesto la homogeneización de los modelos de vida, difuminando en gran medida algunos de los elementos culturales y sociales que caracterizaban anteriormente a las sociedades rurales. Por otra parte, en muchos espacios rurales las actividades económicas han tendido a una notable diversificación. En definitiva, Feria (2004) recuerda que a medida que los procesos urbanos han ido rompiendo los límites de la ciudad tradicional compacta y generando nuevas formas y estructuras de manifestación de dicha ciudad, los académicos, investigadores y responsables de las diferentes políticas públicas han desarrollado todo un conjunto de nociones y conceptos para aprehender y describir la misma: campo urbano, región urbana funcional (Eurostat, 2004), región metropolitana, áreas metropolitanas (Boix, 2007), áreas micropolitanas, aglomeraciones urbanas, distritos metropolitanos, megalópolis, etc.

Las múltiples aproximaciones al análisis de la ruralidad pueden agruparse en tres enfoques (Paniagua y Hoggart, 2002): cuantitativo, cualitativo y de flujos.

- El enfoque cuantitativo asume que el medio rural puede definirse a través de un conjunto de parámetros (observables, medibles y estadísticamente disponibles) que permitan establecer un valor a partir del cual determinar el grado de ruralidad del territorio analizado (habitualmente el municipio), bien mediante un modelo dual (rural versus no rural), bien mediante modelos continuos que van de lo "muy rural" hasta lo "megaurbano".

- El enfoque cualitativo considera que lo rural y lo urbano son realidades subjetivas que percibe la población en función de sus preferencias. Así, frente a la acotación espacial del enfoque cuantitativo, el cualitativo intenta delimitar los procesos, estructuras y percepciones sociales que caracterizan lo rural.

- El enfoque de flujos se asienta en la atracción que la población urbana (habitualmente de las sociedades post-industriales) siente por los espacios rurales. Detrás de esta atracción, se encuentra una idea de lo rural que se asimila con aspectos (en muchas ocasiones idealizados) relativos a relaciones sociales, medio ambiente, salud o modo de vida.

Las dos últimas no resultan aproximaciones operativas para planificar y tomar decisiones políticas, por lo que, tradicionalmente, al igual que hace la Ley 
para el Desarrollo Sostenible del Medio Rural, el criterio utilizado ha sido el cuantitativo. De esta forma, históricamente la discusión se ha centrado en el número de variables que deben utilizarse para medir el grado de ruralidad y en el criterio óptimo para su delimitación. La utilización de un gran número de parámetros ha obligado a los investigadores a recurrir a técnicas de análisis multivariante. Entre los pioneros en su empleo, debemos señalar a Cloke (1977) que, a través del análisis de componentes principales, diseña un Índice de ruralidad para Inglaterra y Gales ${ }^{12}$ y a Bryant $(1973,1974)$ que examina la región de París aunando el análisis factorial y el análisis cluster. Desde entonces, no han cesado los intentos de diferenciar lo rural de lo urbano mediante la construcción de indicadores que midan el grado de ruralidad de estados y municipios en función de condiciones económicas y sociales ${ }^{13}$.

Paralelamente al desarrollo de los análisis multivariantes, son muchas las aproximaciones que, también desde un enfoque estadístico, han tratado de circunscibir esta realidad simplificando los criterios de decisión (reduciendo el número de variables explicativas), siendo el tamaño de la población y la densidad, por su sencillez y disponibilidad, los más habituales. Con este enfoque, uno de los criterios más utilizados es el propuesto por la OCDE (2005 y 2009) que, en función de la densidad de población, considera rurales a los municipios con menos de 150 habitantes por $\mathrm{km}^{2}$. Agregando municipios homogéneos según su densidad genera una tipología de regiones que califica como región predominantemente rural (más del $50 \%$ de la población reside en municipios rurales), región intermedia (entre el 15\% y el 50\%) y región predominantemente urbana (inferior al $15 \%$ ). Si hay algún municipio con más de 200.000 habitantes que representa al menos el $25 \%$ de la población de la región en una zona predominantemente rural, esta zona se reclasificará como intermedia. Si hay algún municipio mayor de 500.000 habitantes que representa al menos el $25 \%$ de la población de la región en una zona intermedia, ésta se reclasificará como predominantemente urbana.

${ }^{12}$ El índice de ruralidad se establece mediante el análisis de componentes principales a partir de 16 variables censales relativas a empleo, población, migración, condiciones de la vivienda, uso del suelo, modelos de desplazamiento diario, distancia a los centros urbanos cercanos. Este índice fue posteriormente recalculado en los trabajos de Cloke y Edwards (1986) y de Harrington y O'Donoghuse (1998).

${ }^{13}$ A modo de ejemplo, podemos citar la definición de rural que realizó para el Reino Unido el Department for Environment Food and Rural Affairs (DEFRA, 2004); la clasificación y tipificación de los municipios de Castilla y León mediante análisis de factores y conglomerados realizada por Fariña, Gordo, Ríos y Rodríguez (2004); el índice creado por la Secretaría Regional Ministerial de Planificación y Coordinación de la Región Metropolitana de Santiago de Chile (SERENI, 2003) o el índice de ruralidad para México que plantean Zamudio, Corona y López (2008). 
La población y la densidad, son también, aunque de forma más simple, los parámetros que Eurostat ha venido empleando desde 1999. Este organismo considera zonas rurales a las no urbanas, siendo estas últimas las que tienen más de 50.000 habitantes y más de 500 habitantes por $\mathrm{km}^{2}$.

En España, no existe una delimitación generalmente aceptada de zonas rurales y urbanas ni a efectos puramente estadísticos ni, lógicamente, a efectos institucionales. Son varios los organismos que intentan superar esta carencia realizando propuestas, siendo el Instituto Nacional de Estadística el pionero en este campo. A partir de parámetros exclusivamente demográficos, tipifica en el Censo de Población de 1981 como zona urbana al conjunto de entidades singulares con más 10.000 habitantes de hecho; zona intermedia a las entidades de 2.001 a 10.000 y zona rural al conjunto de entidades singulares de población con 2.000 o menos habitantes de hecho.

Sin embargo. en el caso español, delimitar las áreas rurales a partir de criterios poblacionales está condicionado por la naturaleza de su división administrativa municipal. Esta estructura, resultado de diferentes procesos históricos, ha configurado un mapa caracterizado por fuertes diferencias entre los municipios (Tabla 1), no sólo entre las distintas Comunidades Autónomas, sino dentro de las mismas. Una de estas diferencias es su extensión, pudiendo encontrar hasta 15 municipios ${ }^{14}$ con menos de $1 \mathrm{~km}^{2}$ y seis ${ }^{15}$ con más de 1.000 $\mathrm{km}^{2}$. Esta diversidad limita la representatividad de la densidad y la población como variables definitorias del tipo de área.

Tabla 1

Distribución de los municipios españolas según su superficie $\left(\mathrm{km}^{2}\right)$

\begin{tabular}{|l|c|c|c|c|c|c|}
\hline & $\begin{array}{c}\mathbf{1} \mathbf{a} \mathbf{1 0} \\
\mathbf{k m}^{2}\end{array}$ & $\mathbf{1 0} \mathbf{a} \mathbf{2 5} \mathbf{~ k m}^{2}$ & $\mathbf{2 5} \mathbf{a} \mathbf{5 0} \mathbf{~ k m}^{2}$ & $\mathbf{5 0} \mathbf{a} \mathbf{1 0 0} \mathbf{~ k m}^{\mathbf{2}}$ & $\begin{array}{c}\mathbf{1 0 0} \mathbf{~ k m}^{\mathbf{2}} \mathbf{y} \\
\text { más }\end{array}$ & $\begin{array}{c}\text { Total } \\
\text { general }\end{array}$ \\
\hline $\mathrm{N}^{\circ}$ municipios & 770 & 2.194 & 2.245 & 1.618 & 1.288 & 8.115 \\
\hline Extensión $\left(\mathrm{km}^{2}\right)$ & 4.932 & 38.106 & 80.786 & 112.998 & 267.450 & 504.273 \\
\hline Población $(2009)$ & 2.535 .521 & 5.430 .990 & 8.587 .902 & 8.943 .804 & 21.247 .590 & 46.745 .807 \\
\hline
\end{tabular}

Fuente: Elaborado a partir del Registro de Entidades Locales.

Recientemente, uno de los intentos más consistentes de separar ambas áreas ha sido el realizado por el Ministerio de Fomento en el Atlas Estadístico de las

${ }^{14}$ Emperador (0,03), Llocnou de la Corona $(0,04)$, Maleján $(0,08)$, Benirredrà $(0,39)$, Puigdàlber $(0,41)$, Geldo $(0,56)$, Beniflá $(0,62)$, Castellfollit de la Roca $(0,67)$, Caldes d'Estrac $(0,74)$, Tavernes Blanques $(0,74)$, Benetússer $(0,78)$, Granja de la Costera, la $(0,83)$, Sant Hipòlit de Voltregà $(0,9)$, Badia del Vallès $(0,93)$ y Palmera $(0,98)$.

15 Cáceres $(1.750,3)$, Lorca $(1.675,2)$, Badajoz $(1.470,4)$, Córdoba $(1.255,2)$, Almodóvar del Campo $(1.208,3)$, Jerez de la Frontera $(1.188,3)$ y Albacete $(1.125,9)$. 
Áreas Urbanas en España ${ }^{16}$. En el correspondiente al año 2009, como norma general considera que las grandes áreas urbanas están integradas por un municipio de al menos 50.000 habitantes más una serie de municipios adyacentes elegidos en función de cuatro tipos de datos: población (tamaño y estructura; densidad; dinámicas demográficas; movilidad laboral; población ocupada según sector económico), vivienda (dinámica en el parque de viviendas; estructura del parque de viviendas según utilización de las mismas; tipología; dinámica de visados de obra; precio de la vivienda), estructura territorial y dinámicas urbanas (observadas a partir de ortofotos, imágenes satélite, usos del suelo, clasificaciones y calificaciones urbanísticas, bases topográficas, documentos de planificación o análisis territorial de Comunidades Autónomas, Diputaciones, Cabildos, Entidades Financieras, etc.) y redes de transportes existentes y planificadas. En total, el Atlas delimita 85 grandes áreas urbanas mayores de 50.000 habitantes, (20 abarcan un sólo término municipal y 65 están compuestas por varios municipios) que agrupan un total de 747 municipios en los que viven más de 30 millones de personas según el Padrón Municipal de Habitantes de 2009. Es decir, el 68\% de la población española vive en el $9,2 \%$ de los municipios. En términos de superficie, el conjunto de estas áreas ocupa tan solo el 9,6\% del territorio nacional. Tipifica, además, pequeñas áreas urbanas, donde incluye, por una parte, los 123 municipios entre 20.000 y 50.000 habitantes no pertenecientes a grandes áreas urbanas, junto con 183 municipios (de los 646 existentes) entre 20.000 y 5.000 habitantes ${ }^{17}$. En resumen, según el Ministerio de Fomento, las áreas no urbanas (rurales) españolas abarcan el $87 \%$ de los municipios (7.059), el $79,8 \%$ del territorio nacional $\left(403.739 \mathrm{~km}^{2}\right)$, donde vive el $19,3 \%$ de la población (9.021.941 habitantes) (Tabla 2).

${ }^{16}$ El primer Atlas Estadístico de las Áreas Urbanas en España corresponde al año 2000 que, a su vez, constituye el segundo de la serie iniciada, a finales de 1998, con la publicación del Atlas Estadístico de la Vivienda en España por el Ministerio de Fomento.

${ }^{17}$ Para establecer cuál de los 646 municipios entre 5.000 y 20.000 habitantes pueden considerarse pequeñas áreas urbanas el Atlas Estadístico de las Áreas Urbanas de España aplica cuatro filtros. En primer lugar, se han tenido en cuenta los 142 cuya población en núcleo supera los 10.000 habitantes. En segundo lugar, se han eliminando 23 municipios que han perdido población entre 1960 y 2009. En tercer lugar, de estos 119 municipios se han eliminado 74 cuyo porcentaje de población activa dedicada al comercio y otros servicios es inferior al porcentaje que a este sector se dedica, por término medio, en los 123 municipios entre $20.000 \mathrm{y}$ 50.000 habitantes no incluidos en las grandes áreas urbanas. Este tercer filtro reduce los municipios a 45. De los 601 municipios excluidos, el cuarto y último filtro recupera 138 municipios, especialmente turísticos, en función de su potencial de acogida, determinado por el alto número de viviendas secundarias y vacías. 
Tabla 2

Características de las zonas rurales

\begin{tabular}{|l|c|c|c|c|c|c|}
\hline \multirow{2}{*}{ Criterios } & \multicolumn{2}{|c|}{ Población } & \multicolumn{2}{c|}{ Municipios } & \multicolumn{2}{c|}{ Superficie } \\
\cline { 2 - 7 } & Habitantes & $\%$ & Número & $\%$ & $\mathbf{K m}^{2}$ & $\%$ \\
\hline OCDE & 10.930 .307 & $23,4 \%$ & 7.002 & $86,3 \%$ & 457.034 & $90,3 \%$ \\
\hline Eurostat & 26.266 .182 & $56,2 \%$ & 8.009 & $98,7 \%$ & 496.930 & $98,2 \%$ \\
\hline INE & 2.821 .282 & $6,0 \%$ & 5.789 & $52,3 \%$ & 264.702 & $71,4 \%$ \\
\hline Atlas & 9.021 .941 & $19,3 \%$ & 7.059 & $87,0 \%$ & 403.739 & $79.8 \%$ \\
\hline
\end{tabular}

Fuente: Elaboración propia.

Frente a este criterio tan estricto, el de Eurostat sólo permite encontrar 145 municipios españoles que cumplen la primera condición para ser considerados urbanos (más de 50.000 habitantes) aunque, de éstos, 41 no cumplen la segunda (500 habitantes por $\mathrm{km}^{2}$ ). En otras palabras, excepto 104 municipios, el resto son calificables de rurales, lo que equivale al $98,2 \%$ del territorio nacional. De hecho, conforme a este criterio, varias capitales de provincia serían catalogadas como zona rural no urbana ${ }^{18}$. Paralelamente, encontramos localidades que en función de su población tendrían un claro carácter urbano, pero como están ubicadas en un municipio muy extenso, incumplen el criterio de la densidad, siendo catalogadas como rurales. Además, existen municipios que, aunque rurales, se localizan junto a espacios urbanos, siendo un apéndice de un núcleo de mayor entidad. Si comparamos los cuatro criterios indicados (Tabla 2), comprobamos que, mientras que con el establecido por el INE, sólo el 6\% de la población española viviría en municipios rurales, con el de Eurostat, este porcentaje asciende al 56,2\%. Pero, incluso, de los 5.789 municipios que según el INE son rurales, 100 no lo son según el criterio de la OCDE.

La Ley 45/2007 no asume ninguno de estos cuatro criterios (OCDE, Eurostat, INE y Atlas), considerando que las zonas rurales se componen por agregación de municipios rurales ${ }^{19}$ que posean una población inferior a 30.000 habitantes y una densidad inferior a los 100 habitantes por $\mathrm{km}^{2}$, o en su caso de entidades locales menores. Excepcionalmente, se permite incluir en una zona rural un municipio que no cumpla alguno de los dos criterios citados, cuando la homogeneidad y funcionalidad de la zona así lo exija. Corresponde a las Comunidades Autónomas (art. 9) delimitar y calificar como tales a las zonas rurales en tres tipos (art. 10):

${ }^{18}$ Por ejemplo, en Castilla y León sólo es posible encontrar nueve municipios con más de 50.000 habitantes (las nueve capitales de provincia, excepto Soria, más Ponferrada) de los cuales, Ávila, Segovia, Zamora y Ponferrada no alcanzan el criterio de la densidad.

${ }^{19}$ Se considera municipio rural de pequeño tamaño aquel que posea una población residente inferior a los 5.000 habitantes y esté integrado en el medio rural. 
a) Zonas rurales a revitalizar: aquellas con escasa densidad de población, elevada significación de la actividad agraria, bajos niveles de renta y un importante aislamiento geográfico o dificultades de vertebración territorial.

b) Zonas rurales intermedias: aquellas de baja o media densidad de población, con un empleo diversificado entre el sector primario, secundario y terciario, bajos o medios niveles de renta y distantes del área directa de influencia de los grandes núcleos urbanos.

c) Zonas rurales periurbanas: aquellas de población creciente, con predominio del empleo en el sector terciario, niveles medios o altos de renta y situadas en el entorno de las áreas urbanas o áreas densamente pobladas.

A su vez, se consideran zonas prioritarias (o de primer nivel de prioridad) a todas aquellas a revitalizar, además de las zonas intermedias en las que más del $80 \%$ de la población viva en municipios con menos de 5.000 habitantes o en las que la superficie de la Red Natura 2000 más la de los municipios con menos de 5.000 habitantes represente más del $80 \%$ de la zona.

Tal y como se ha señalado, el principal instrumento de aplicación de la Ley 45/2007 es, como se indica en su exposición de motivos, el Programa de Desarrollo Rural Sostenible para el periodo 2010-2014. En éste se consideran 219 zonas rurales catalogadas en la forma que recoge la Tabla 3, de las cuales 108 son prioritarias.

Tabla 3

Distribución de las zonas rurales españolas

\begin{tabular}{|l|c|c|c|c|}
\hline & $\begin{array}{c}\text { Zonas rurales } \\
\text { a revitalizar }\end{array}$ & $\begin{array}{c}\text { Zonas rurales } \\
\text { intermedias }\end{array}$ & $\begin{array}{c}\text { Zonas rurales } \\
\text { periurbanas }\end{array}$ & Total \\
\hline $\mathbf{1}^{\text {er }}$ nivel (prioritarias) & 105 & 3 & - & 108 \\
\hline $\mathbf{2}^{\mathbf{0}}$ nivel & - & 51 & - & 51 \\
\hline $\mathbf{3}^{\text {er }}$ nivel & - & 18 & 15 & 33 \\
\hline Sin prioridad & - & 12 & 15 & 27 \\
\hline Total & 105 & 84 & 30 & 219 \\
\hline
\end{tabular}

Fuente: Elaborado a partir del Programa de Desarrollo Rural Sostenible 2010-2014.

Esta clasificación presenta serios inconvenientes para su utilización operativa. El principal de ellos radica en que varias zonas rurales se delimitan, 
además de por municipios, por entidades locales menores, entidades singulares de población e, incluso, polígonos catastrales ${ }^{20}$.

Con el fin de alcanzar la máxima operatividad, en el presente trabajo, se ha utilizado el municipio como unidad básica de información. Para ello se han realizado los siguientes ajustes:

- Un municipio se estimará no rural y, por tanto, urbano, cuando sólo se haya considerado rural alguna pedanía, entidad local menor o polígono catastral. Las exclusiones han supuesto que en 19 de las zonas, inicialmente sólo rurales, se hayan contabilizado 67 municipios de carácter urbano $^{21}$.

- Se han excluido los territorios navarros (facerías) que pertenecen en común a varios municipios.

- Un municipio se cataloga como rural cuando de él se excluyen determinadas localidades ${ }^{22}$, excepto si el municipio en conjunto tiene más de 30.000 habitantes o en la excepción se indica explícitamente la existencia de una zona urbana, en cuyo caso se considera urbano ${ }^{23}$.

- Tras aplicar este filtro, la distribución de los municipios españoles que utilizaremos en nuestro análisis se recoge en la Tabla 4.

${ }^{20}$ Por ejemplo, en el municipio de Cáceres sólo se consideran las entidades locales menores de Rincón de Ballesteros y Valdesalor pertenecientes a la Zona Rural de Comarca de Trujillo, Sierra de Montánchez y Zona Centro, por lo que, de considerar todo el término municipal como zona rural, estaríamos catalogando a la capital cacereña como zona rural a revitalizar de forma prioritaria. En el municipio leridano de Les Borges Blanques, sólo se considera zona rural a los polígonos catastrales 1 , del 5 al 10 y del 12 al 22, mientras que en el municipio de Tremp, sólo 28 pedanías están catalogadas como zona rural.

${ }^{21}$ En 5 zonas rurales a revitalizar (Comarca de Trujillo, Sierra de Montánchez y Zona Centro; Valle del Alagón, Rivera de Fresnedosa y Riberos del Tajo; Noreste de Cádiz y Los Alcornocales; Garrigues y Segrià sureste; Pallars Jussà, Pallars Sobirà y Alta Ribagorça; Sierras Occidentales de Málaga y Salamanca Sureste) se han considerado siete municipios urbanos: Cáceres, Plasencia, Jerez de la Frontera, Borges Blanques (Les), Tremp, Ronda y Béjar. En 10 de las 84 zonas rurales intermedias se han excluido a 50 municipios, una parte importante de ellos ubicados en la zona rural de Tenerife (19) y en la de Gran Canaria (12). Paralelamente, de 4 zonas rurales periurbanas se han excluido 10 municipios por considerarse urbanos.

${ }^{22}$ Por ejemplo, el Programa de Desarrollo Rural Sostenible califica de rural, a excepción de un número determinado de parroquias. a los municipios asturianos de Proaza, Carreño y Gozón (en la Zona Rural Occidente Asturiano) y Colunga Villaviciosa, Aller, Laviana, Lena, Llanes, Ribadesella (en la Zona Rural Oriente Asturiano).

${ }^{23}$ Es el caso de Calvià, Eivissa, Manacor, Ronda, Llucmajor, Marratxí y Santa Eulalia del Río, Ciutadella de Menoría, Maó, Sant Josep de Sa Talaia, Sant Antoni de Portmany, Alcúdia, Pollença, Son Servera y Es Castell. 
Tabla 4

Distribución de los municipios españoles

\begin{tabular}{|l|c|c|c|c|c|c|}
\hline \multicolumn{1}{|c|}{ Prioridad } & $\begin{array}{c}\text { Municipios } \\
\text { rurales a } \\
\text { revitalizar }\end{array}$ & $\begin{array}{c}\text { Municipios } \\
\text { rurales } \\
\text { intermedios }\end{array}$ & $\begin{array}{c}\text { Municipios } \\
\text { rurales } \\
\text { periurbanos }\end{array}$ & $\begin{array}{c}\text { Municipios } \\
\text { urbanos }\end{array}$ & Total & $\%$ \\
\hline $1^{\text {er }}$ nivel & 3.916 & 15 & - & - & 3.931 & $48,4 \%$ \\
\hline $2^{\circ}$ nivel & - & 1.442 & - & - & 1.442 & $17,8 \%$ \\
\hline $3^{\text {er }}$ nivel & - & 386 & 465 & - & 851 & $10,5 \%$ \\
\hline Sin prioridad & - & 223 & 266 & 1.401 & 1.890 & $23,3 \%$ \\
\hline Total & $\mathbf{3 . 9 1 6}$ & $\mathbf{2 . 0 6 6}$ & $\mathbf{7 3 1}$ & $\mathbf{1 . 4 0 1}$ & $\mathbf{8 . 1 1 4}$ & $\mathbf{1 0 0 , 0 \%}$ \\
\hline $\mathrm{N}^{\circ}$ municipios & $48,3 \%$ & $25,5 \%$ & $9,0 \%$ & $17,3 \%$ & $100,0 \%$ & - \\
\hline Población & $6,6 \%$ & $11,5 \%$ & $5,1 \%$ & $76,8 \%$ & $100,0 \%$ & - \\
\hline Territorio & $50,1 \%$ & $26,3 \%$ & $6,8 \%$ & $16,8 \%$ & $100,0 \%$ & - \\
\hline
\end{tabular}

Fuente: Elaborado a partir del Programa de Desarrollo Rural Sostenible 2010-2014.

De acuerdo con estos criterios, el 82,7\% de los municipios españoles se localizan en zona rural, y, uno de cada dos (3.916) en zona rural a revitalizar de forma prioritaria. Sin embargo, estas cifras distan mucho de ser homogéneas en el conjunto de España. Así, por ejemplo, en cuatro Comunidades Autónomas el número de municipios a revitalizar de forma prioritaria supera el 70\%: Asturias (85,9\%), Castilla y León (74,6\%), Aragón (73,9\%) y Extremadura (71,9\%). Paralelamente, en las tres últimas los municipios urbanos no llegan al 2,5\% del total. En el conjunto de España, aunque el número de municipios urbanos sólo representa el $17,3 \%$ del total y el $16,8 \%$ de la superficie, concentran el $76,8 \%$ de la población, lo que significa, desde otra perspectiva, que en las zonas rurales sólo vive uno de cada cuatro españoles. Es más, las zonas rurales a revitalizar de forma prioritaria que ocupan la mitad del territorio nacional, sólo recogen el $6,6 \%$ de la población, destacando por encima de esta cifra Extremadura $(39,5 \%)$, Asturias $(26,0 \%)$ y Castilla y León $(24,7 \%)$.

Por otra parte, la Ley 45/2007 considera 3.931 municipios de primer nivel (prioritarios), aunque 325 de ellos se localizan en Comunidades en las que no es de aplicación el Sistema Nacional de Incentivos Regionales: Cataluña (140), Navarra (90) y La Rioja (95). Además, establece 2.361 municipios de carácter prioritario no considerados directamente como tales en los Reales Decretos de delimitación de las zonas de promoción económica de las respectivas Comunidades Autónomas, si bien indirectamente, en todos ellos se señala que se tendrán en cuenta las zonas rurales prioritarias que se establezcan en aplicación de la Ley 45/2007 para el desarrollo sostenible del medio rural. 


\section{LOS PROYECTOS INCENTIVADOS TRAS LA APROBACIÓN DE LA LEY 45/2007}

El presente epígrafe tiene por finalidad analizar si en el tiempo transcurrido desde la aprobación de la Ley 45/2007 hasta la entrada en vigor del Programa de Desarrollo Rural Sostenible se aprecia la existencia de discriminación positiva hacia las zonas rurales. Para ello, en primer lugar, se comparan los incentivos concedidos en cada Comunidad Autónoma entre 2000 y 2002 con los concedidos entre 2008 y 2010. En segundo lugar, se contrasta si ha existido un cambio significativo en las características de los incentivos concedidos en ambos periodos. Por último, el análisis se centra en determinar si existen diferencias significativas entre los proyectos incentivados dependiendo de la zona en que se localizan.

Previamente, hay que recordar que la Ley 50/1985 de Incentivos Regionales nace 22 años antes que la Ley 45/2007 y, por tanto, que entre sus objetivos no figura expresamente el desarrollo rural. De hecho, la palabra "rural" no se cita ni en el articulado de la Ley, ni en el primer Reglamento que la desarrolla, ni en los iniciales Reales Decretos de delimitación de las diferentes zonas (excepto cuando se menciona el turismo rural como sector a incentivar). Es más, la Ley 50/1985, antes de estar al servicio del desarrollo rural, ha sido el instrumento sobre el que se ha sustentado el Programa Operativo de Mejora de la Competitividad y Desarrollo del Tejido Productivo para el periodo 2000-2006 en el que, tampoco, las zonas rurales han tenido un tratamiento diferenciado. No obstante, sí se citan explícitamente el objetivo de "repartir más equilibradamente las actividades económicas" sobre el territorio y "reforzar el potencial de desarrollo endógeno de las regiones". Si, junto a estas referencias, tenemos en cuenta las condiciones particulares que se establecen para los municipios calificados de prioritarios, resulta innegable que la orientación municipal está presente en la definición y desarrollo de la Ley. No podía ser de otra forma, pues, en última instancia, el crecimiento de una región o zona promocionable es el reflejo de las mejoras producidas en los territorios y municipios que la conforman (Moyano, Aleixandre y Ogando, 2006).

Dado el escaso tiempo que ha pasado desde la entrada en vigor del primer Programa de Desarrollo Rural Sostenible, resulta prematuro tratar de evaluar este instrumento. En el presente epígrafe y en el siguiente se analiza si en los tres años transcurridos (2008 a 2010) desde la aprobación de la Ley 45/2007 hasta al entrada en vigor del citado Programa se vislumbra un cambio de tendencia que permita aventurar algún tipo de discriminación positiva hacia las zonas rurales, en general, y hacia aquellas a revitalizar, en particular. Comenzaremos, para ello, comparando los incentivos concedidos en cada Comunidad Autónoma durante el primer trienio (2000-2002) de aplicación de la Ley de Incentivos Regionales al servicio del Programa Operativo de Mejora de 
la Competitividad con los concedidos durante los tres primeros años (20082010) como instrumento de desarrollo rural, al servicio de Ley para el Desarrollo Sostenible del Medio Rural. A tal fin se ha elaborado la Tabla 5. La lectura de los datos permite destacar los siguientes hechos:

- La enorme reducción en el número de concesiones en el segundo trienio respecto al primero. Los incentivos decrecen un $67,3 \%$, pasando de 1.917 a 627. Además, la experiencia demuestra que una parte importante de estas concesiones resultarán fallidas, no llegando a ser efectivas. Dado el escaso periodo de tiempo transcurrido, sólo 5 de las 627 concesiones no se han materializado. A la luz de la experiencia acumulada, es previsible que esta cifra se incremente de manera significativa con el paso del tiempo. De hecho, esta cantidad se eleva a $503(26,2 \%)$ de las otorgadas entre 2000 y 2002. Además de ellas, otras $400(20,9 \%)$ han registrado algún tipo de modificación de las condiciones iniciales ${ }^{24}$.

Tabla 5

Incentivos concedidos en las Comunidades Autónomas al amparo de la Ley 50/1985

\begin{tabular}{|c|c|c|c|c|c|c|c|c|c|c|}
\hline \multirow{3}{*}{$\begin{array}{c}\text { Comunidades } \\
\text { Autónomas }\end{array}$} & \multicolumn{5}{|c|}{2000 a 2002} & \multicolumn{5}{|c|}{2008 a 2010} \\
\hline & \multicolumn{2}{|c|}{ Total } & \multicolumn{3}{|c|}{ En zonas rurales } & \multicolumn{2}{|c|}{ Total } & \multicolumn{3}{|c|}{ En zonas rurales } \\
\hline & $\mathbf{N}^{\circ}$ & $\%$ & $\mathbf{N}^{\circ}$ & $\%$ & $\%$ s/ total & $\mathbf{N}^{\circ}$ & $\%$ & $\mathbf{N}^{\circ}$ & $\%$ & $\%$ s/ total \\
\hline Andalucía & 484 & $25,2 \%$ & 237 & $24,5 \%$ & $49,0 \%$ & 115 & $18,3 \%$ & 51 & $13,9 \%$ & $44,3 \%$ \\
\hline Aragón & 61 & $3,2 \%$ & 41 & $4,2 \%$ & $67,2 \%$ & 27 & $4,3 \%$ & 21 & $5,7 \%$ & $77,8 \%$ \\
\hline Asturias & 89 & $4,6 \%$ & 10 & $1,0 \%$ & $11,2 \%$ & 42 & $6,7 \%$ & 10 & $2,7 \%$ & $23,8 \%$ \\
\hline Canarias & 95 & $5,0 \%$ & 3 & $0,3 \%$ & $3,2 \%$ & 23 & $3,7 \%$ & 1 & $0,3 \%$ & $4,3 \%$ \\
\hline Cantabria & 33 & $1,7 \%$ & 2 & $0,2 \%$ & $6,1 \%$ & 5 & $0,8 \%$ & 2 & $0,5 \%$ & $40,0 \%$ \\
\hline Castilla y León & 327 & $17,1 \%$ & 212 & $21,9 \%$ & $64,8 \%$ & 122 & $19,5 \%$ & 94 & $25,7 \%$ & $77,0 \%$ \\
\hline Castilla-La Mancha & 130 & $6,8 \%$ & 50 & $5,2 \%$ & $38,5 \%$ & 36 & $5,7 \%$ & 14 & $3,8 \%$ & $38,9 \%$ \\
\hline Ceuta & 1 & $0,1 \%$ & - & - & - & 2 & $0,3 \%$ & - & - & - \\
\hline Com. Valenciana & 334 & $17,4 \%$ & 184 & $19,0 \%$ & $55,1 \%$ & 26 & $4,1 \%$ & 11 & $3,0 \%$ & $42,3 \%$ \\
\hline Extremadura & 101 & $5,3 \%$ & 66 & $6,8 \%$ & $65,3 \%$ & 113 & $18,0 \%$ & 86 & $23,5 \%$ & $76,1 \%$ \\
\hline Galicia & 202 & $10,5 \%$ & 158 & $16,3 \%$ & $78,2 \%$ & 99 & $15,8 \%$ & 73 & $19,9 \%$ & $73,7 \%$ \\
\hline Melilla & - & - & - & - & - & - & - & - & - & 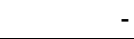 \\
\hline Murcia & 60 & $3,1 \%$ & 6 & $0,6 \%$ & $10,0 \%$ & 17 & $2,7 \%$ & 3 & $0,8 \%$ & $17,6 \%$ \\
\hline Total & 1.917 & $100,0 \%$ & 969 & $100,0 \%$ & $50,5 \%$ & 627 & $100,0 \%$ & 366 & $100,0 \%$ & $58,4 \%$ \\
\hline
\end{tabular}

Fuente: Elaboración propia.

${ }^{24}$ De forma ilustrativa podemos recordar que una tercera parte de los 10.009 proyectos inicialmente aprobados en el marco del Programa Operativo de Mejora de la Competitividad y Desarrollo del Tejido Productivo 2000-2006, no se han materializado por haber decaído, caducado o incumplido gravemente las condiciones estipuladas. Estas incidencias han supuesto 7.600 millones de euros menos de inversión que, de haberse ejecutado, hubieran representado 58.584 empleos adicionales (Moyano y Aleixandre, 2009). 
- La reducción en el número de concesiones ha sido generalizada, aunque especialmente intensa en Andalucía (369), Comunidad Valenciana (308) y Castilla y León (205). Únicamente Extremadura y, testimonialmente, Ceuta ven incrementar el número de inversiones incentivadas (12 y 1 , respectivamente).

- A pesar de que los incentivos a la inversión han pasado a ser un instrumento al servicio del desarrollo rural, el número de los concedidos en este medio se reduce un $62,2 \%$, pasando de 969 a 366, siendo las tres Comunidades antes citadas las que sufren la mayor disminución. En el caso de la Comunidad Valenciana esta pérdida supone el 94\% (de 184 a 11) y el $78,5 \%$ en Andalucía (de 237 a 51). En ambas regiones (junto con Galicia), el porcentaje de reducción es más intenso que el experimentado en el medio urbano. Sin embargo, para el conjunto de España, las subvenciones en los municipios urbanos decrecen un $72,5 \%$.

- La diferente intensidad con que se produce la minoración en el número de incentivos en cada Comunidad ha provocado un cambio importante en la distribución territorial. Así, mientras que entre 2000 y 2002, una de cada cuatro concesiones se realizó a empresas andaluzas $(25,2 \%)$, a partir del año 2008 este porcentaje se reduce al 18,3\%. Especialmente significativa resulta la pérdida de peso relativo de la Comunidad Valenciana y el aumento de Extremadura ${ }^{25}$. De hecho, esta última, junto con Andalucía, Castilla y León y Galicia han concentrado el $71,6 \%$ de las concesiones desde 2008 y el $83,1 \%$ de las otorgadas en el medio rural. Es más, prácticamente la mitad de las subvenciones a la inversión realizada por empresas localizadas en zonas rurales se ubican en Extremadura $(23,5 \%)$ o en Castilla y León $(25,7 \%)$.

Antes de proceder a analizar si con la entrada en vigor de la Ley 45/2007 para el desarrollo sostenible del medio rural se ha producido un cambio significativo en las características de los incentivos concedidos, debemos averiguar si junto a la drástica reducción en el número de incentivos se observan modificaciones en sus características fundamentales (inversión real por proyecto $^{26}$, empleo creado por proyecto e inversión por empleo). Se trata de contrastar mediante el procedimiento Anova la siguiente hipótesis ${ }^{27}$ :

${ }^{25}$ Hay que recordar que en el periodo de programación de la política de cohesión 2007-2013, Extremadura es considerada Región Convergencia mientras que la Comunidad Valenciana se considera Región Phasing in.

${ }^{26}$ Euros de 2008 calculados con el Índice de Precios Industriales del INE.

${ }^{27}$ Como se ha comentado, el objetivo del análisis de la varianza es contrastar la hipótesis de que varias medias son iguales. Este contraste se concreta del siguiente modo. $\mathrm{H}_{\mathrm{o}}: \alpha_{1}=\alpha_{2}=\alpha_{3}=\ldots=\alpha_{\kappa} ; \quad \mathrm{H}_{1}:$ alguna $\alpha_{\mathrm{i}} \neq \alpha_{\mathrm{j}}$ 
Ho: los proyectos incentivados a partir de 2008 tienen las mismas características en cuanto a su tamaño que los incentivados entre 2000 y 2002

La Tabla 6 describe el valor que alcanzan estas características en cada uno de los dos periodos considerados. Se recoge, además, el nivel critico o nivel de significación observado (sig) del procedimiento Anova a partir del cual aceptar o rechazar la hipótesis de igualdad de medias ${ }^{28}$ y el nivel de significación (sig.) del test de Levene que permite evaluar la homogeneidad de varianzas ${ }^{29}$.

Tabla 6

Características de los proyectos incentivados entre 2000-2002 y 2008-2010

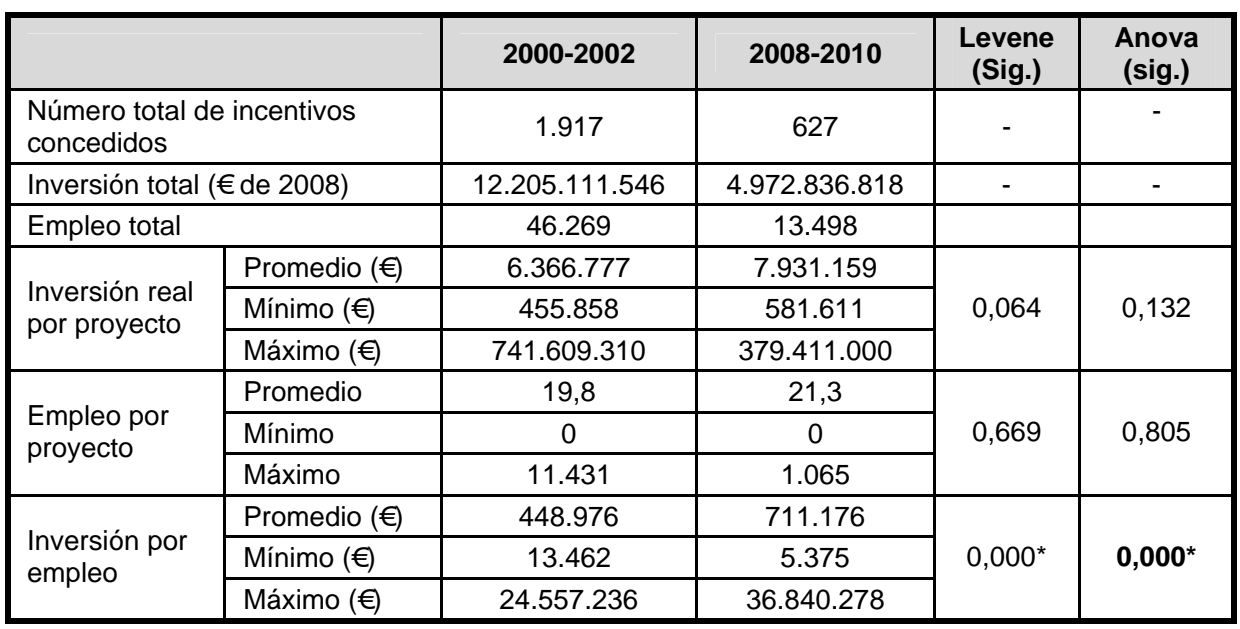

(*) Nivel de significación: 0,05.

Fuente: Elaboración propia.

Los datos permiten aceptar la hipótesis nula tanto para la inversión media $(p=0,132)$ como para el empleo creado por proyecto $(p=0,805)$, es decir, los proyectos subvencionados presentan características similares respecto a su tamaño, bien se mida éste a través de la inversión generada, bien por el empleo creado. En ambas variables se cumple, además, el supuesto de homocedasticidad o igualdad de varianzas contrastado mediante el estadístico de Levene ( $\mathrm{p}=0,064$ y $\mathrm{p}=0,669)$. No ocurre lo mismo respecto a la inversión

${ }^{28}$ El nivel de significación muestra la probabilidad de rechazar la hipótesis nula y que sea cierta. Cuando el valor del nivel critico es menor que 0,05 se rechaza la hipótesis nula (de igualdad de medias) $\mathrm{y}$, por tanto, se puede afirmar que las medias de las distintas poblaciones no son iguales.

${ }^{29}$ Cuando el valor del nivel critico es menor que 0,05 se rechaza la hipótesis de homogeneidad de varianzas (homocedasticidad). 
requerida por empleo creado, donde ambos valores de significación son nulos. Este hecho está motivado, exclusivamente, por la disparidad mostrada en las zonas rurales intermedias, lo que se confirma cuando se realizan comparaciones bilaterales entre los dos periodos mediante la prueba $\mathrm{T}$.

La Tabla 7 recoge los resultados de estas comparaciones, indicándose en la parte izquierda de las celdas (cursiva), el nivel de significación de la prueba de Levene para la igualdad de varianzas, y en la derecha el nivel de significación de la prueba $\mathrm{T}$ para la igualdad de medias, dependiendo este valor de si previamente se confirma o no la igualdad de varianzas. Los valores constatan que, salvo en la inversión $(\mathrm{p}=0,014) \mathrm{y}$, especialmente, en la inversión por empleo $(\mathrm{p}=0,001)$, en las zonas rurales intermedias, los proyectos incentivados presentan las mismas características en ambos periodos.

Tabla 7

Similitud en las características de los proyectos incentivados en cada zona entre 2000-2002 y $2008-2010$

\begin{tabular}{|c|c|c|c|c|c|c|c|c|c|}
\hline & \multicolumn{6}{|c|}{ Zonas rurales } & \multirow{2}{*}{\multicolumn{2}{|c|}{$\begin{array}{l}\text { Zonas } \\
\text { Urbanas }\end{array}$}} \\
\hline & & \multicolumn{2}{|c|}{ A revitalizar } & \multicolumn{2}{|c|}{ Intermedias } & \multicolumn{2}{|c|}{ Periurbanas } & & \\
\hline \multirow{3}{*}{$\begin{array}{c}\text { Prueba T } \\
\text { (niveles de sig. } \\
\text { bilaterales) }\end{array}$} & Inversión & 0,270 & 0,214 & 0,000 * & $0,014^{*}$ & 0,000 & 0,110 & 0,281 & 0,357 \\
\hline & Empleo & 0,715 & 0,576 & 0,004 & 0,151 & 0,000 & 0,266 & 0,459 & 0,620 \\
\hline & Inv. I empleo & 0,207 & 0,281 & $0,000^{*}$ & $0,001^{*}$ & 0,505 & 0,258 & 0,000 & 0,050 \\
\hline
\end{tabular}

(*) Nivel de significación: 0,05.

Fuente: Elaboración propia.

Asumiendo que no existen diferencias estadísticamente significativas entre ambos periodos, la siguiente cuestión a contrastar es si los proyectos subvencionados a partir de 2008, muestran características diferenciadas en función de si se localizan en zonas rurales o urbanas. Las características consideradas se sintetizan en la Tabla 8. Entre 2008 y 2010 se han subvencionado 627 proyectos con una inversión prevista cercana a 5 millones de euros que lleva asociada la creación de 13.498 empleos. En conjunto, sólo el $47,5 \%$ de la inversión se localiza en áreas rurales, a pesar de que en ellas se ubican el $58,4 \%$ de los proyectos y está previsto crear el $57 \%$ del empleo. De forma más específica, en las zonas rurales con mayor prioridad en la captación de incentivos sólo se concentra el $14 \%$ de la inversión a pesar de contar con el $23,1 \%$ de los proyectos. Obviamente, tras estas cifras se esconde una realidad caracterizada por el hecho de que los proyectos de las zonas urbanas son, en general, de mayor dimensión que los de las zonas rurales. 


\section{Tabla 8}

Características de los proyectos incentivados entre 2008 y 2010 según tipo de zona

\begin{tabular}{|c|c|c|c|c|c|c|c|}
\hline & & \multicolumn{4}{|c|}{ Zonas rurales } & \multirow{2}{*}{$\begin{array}{l}\text { Zonas } \\
\text { Urbanas }\end{array}$} & \multirow[b]{2}{*}{ Total } \\
\hline & & $\underset{\text { revitalizar }}{\mathrm{A}}$ & Intermedias & Periurbanas & Total & & \\
\hline \multirow{2}{*}{ Incentivos } & Número & 145 & 184 & 37 & 366 & 261 & 627 \\
\hline & $\%$ & $23,1 \%$ & $29,3 \%$ & $5,9 \%$ & $58,4 \%$ & $41,6 \%$ & $100,0 \%$ \\
\hline \multirow{2}{*}{ Inversión } & $€(2008)$ & 696.150 & 1.194 .606 & 470.356 & 2.361 .112 & 2.611 .726 & 4.972 .838 \\
\hline & $\%$ & $14,0 \%$ & $24,0 \%$ & $9,5 \%$ & $47,5 \%$ & $52,5 \%$ & $100,0 \%$ \\
\hline \multirow{2}{*}{ Empleo } & Número & 2.104 & 3.752 & 1.839 & 7.695 & 5.803 & 13.498 \\
\hline & $\%$ & $15,6 \%$ & $27,8 \%$ & $13,6 \%$ & $57,0 \%$ & $43,0 \%$ & $100,0 \%$ \\
\hline \multicolumn{2}{|c|}{$\mathrm{N}^{\circ}$ total de incentivos } & 145 & 184 & 37 & 366 & 261 & 627 \\
\hline \multicolumn{2}{|c|}{$\mathrm{N}^{0}$ total de municipios } & 3.916 & 2.066 & 731 & 6.713 & 1.401 & 8.114 \\
\hline \multicolumn{2}{|c|}{ Municipios incentivados } & 111 & 123 & 26 & 260 & 115 & 375 \\
\hline \multicolumn{2}{|c|}{$\%$ de municipios incentivados } & $2,8 \%$ & $6,0 \%$ & $3,6 \%$ & $3,9 \%$ & $8,2 \%$ & $4,6 \%$ \\
\hline \multicolumn{2}{|c|}{$\mathrm{N}^{\circ}$ de incentivos por municipio } & 1,3 & 1,5 & 1,4 & 1,4 & 2,3 & 1,7 \\
\hline \multicolumn{2}{|l|}{ Población total } & 3.084 .349 & 5.372 .517 & 2.399 .484 & 10.856 .350 & 35.889 .457 & 46.745 .807 \\
\hline \multicolumn{2}{|c|}{ Densidad (Hab. por km²) } & 12,2 & 40,5 & 69,8 & 25,9 & 423,2 & 92,7 \\
\hline \multicolumn{2}{|c|}{ Población municipios incentivados } & 428.368 & 1.002 .600 & 200.197 & 1.631 .165 & 10.135 .769 & 11.766 .934 \\
\hline \multicolumn{2}{|c|}{ Densidad Munic. Incentivados } & 22,5 & 73,3 & 69,2 & 45,8 & 309,6 & 172,2 \\
\hline \multicolumn{2}{|c|}{ Población / incentivos } & 13.063 & 14.959 & 14.231 & 14.077 & 22.696 & 16.720 \\
\hline
\end{tabular}

Fuente: Elaboración propia.

Para determinar si existen diferencias significativas entre los proyectos dependiendo de la zona de localización, comenzamos contrastando la igualdad de medias en el caso de la inversión. Para evitar la distorsión que supone incluir en una sola zona rural territorios tan heterogéneos, el análisis se ceñirá a las cuatro zonas consideradas (tres rurales y una urbana), obviando la comparación de la zona rural total con la zona urbana total. Es decir, la hipótesis nula se concreta de la siguiente forma:

Ho: los proyectos incentivados a partir de 2008 realizan la misma inversión independientemente de la zona en que se localizan

La Tabla 9 muestra que el valor del nivel crítico $(p=0,023)$ es inferior a 0,05 , por lo que podemos rechazar la hipótesis de igualdad de medias y concluir que en las cuatro zonas analizadas, los proyectos no tienen la misma inversión media. Además, esta circunstancia se acompaña con heterocedasticidad de las varianzas ( $\mathrm{p}$-valor del estadístico de Levene $=0,000$ ). No obstante, si repetimos el procedimiento excluyendo del análisis la zona urbana, los contrastes se revelan significativos para aceptar tanto la igualdad de varianzas $(\mathrm{p}=0,198)$ como la de medias $(\mathrm{p}=0,523)$. Es decir, dentro de las áreas rurales no existen diferencias significativas en el tamaño de los proyectos medidos por su volumen de inversión. Este hecho se confirma cuando se realizan comparaciones bilaterales entre zonas mediante la prueba T. Los contrastes (Tabla 9) revelan 
que la única diferencia significativa se produce entre las zonas rurales a revitalizar y las urbanas $(\mathrm{p}=0,004)$ y que el mayor grado de semejanza se observa entre estas últimas y las periurbanas $(\mathrm{p}=0,568)$.

Tabla 9

Inversión por proyecto incentivado según tipo de zona entre 2008 y 2010

\begin{tabular}{|c|c|c|c|c|c|c|c|c|}
\hline & & \multicolumn{5}{|c|}{ Zonas rurales } & \multirow{2}{*}{\multicolumn{2}{|c|}{$\begin{array}{l}\text { Zonas } \\
\text { Urbanas }\end{array}$}} \\
\hline & & A revitalizar & Interr & nedias & Periu & banas & & \\
\hline \multirow{3}{*}{ Descriptivo } & Promedio $(€)$ & 4.801 .034 & 6.49 & 2.422 & 12.71 & 2.314 & 10.00 & 5.612 \\
\hline & Máximo (€) & 45.833 .388 & 63.8 & 7.551 & 129.6 & 3.579 & 379.41 & 1.000 \\
\hline & Mínimo (€) & 603.641 & 605 & .540 & 880 & 543 & 581. & 611 \\
\hline \multicolumn{2}{|c|}{ Levene (significación) } & \multicolumn{7}{|c|}{$0,000 *$} \\
\hline \multicolumn{2}{|c|}{ Anova (significación) } & \multicolumn{7}{|c|}{$0,023^{*}$} \\
\hline \multicolumn{2}{|c|}{ Levene (sig.) zonas rurales } & \multicolumn{5}{|c|}{0,198} & & \\
\hline \multirow{2}{*}{\multicolumn{2}{|c|}{ Anova (sig.) zonas rurales }} & \multicolumn{5}{|c|}{0,523} & & \\
\hline & & A revitalizar & Inter & nedias & Periu & banas & Urba & nas \\
\hline \multirow{3}{*}{$\begin{array}{l}\text { Prueba T } \\
\text { (niveles de } \\
\text { sig. } \\
\text { bilaterales) }\end{array}$} & A revitalizar & - & $0,002^{*}$ & 0,090 & $0,000^{*}$ & 0,057 & $0,001^{*}$ & $0,004^{*}$ \\
\hline & Intermedia & - & \multicolumn{2}{|c|}{-} & $0,002 *$ & 0,134 & $0,021^{*}$ & 0,060 \\
\hline & Periurbana & - & \multicolumn{2}{|c|}{-} & \multicolumn{2}{|c|}{-} & 0,648 & 0,568 \\
\hline
\end{tabular}

(*) Nivel de significación: 0,05.

Fuente: Elaboración propia.

La segunda característica de los proyectos que debemos comparar es el empleo generado por cada proyecto subvencionado. Esta comparación se realiza a través del contraste de la siguiente hipótesis:

Ho: los proyectos incentivados a partir de 2008 generan el mismo empleo independientemente de la zona en que se localizan

Al igual que para la inversión, el contraste de la igualdad de medias para el empleo creado por proyecto (Tabla 10) proporciona un bajo nivel de significación $(\mathrm{p}=0,018)$ que obliga a rechazar la hipótesis nula. Sin embargo, también para esta variable, el origen del rechazo nace de la desigualdad que se produce entre el empleo creado por los proyectos localizados en zonas urbanas y los ubicados en zonas rurales a revitalizar $(p=0,027)$. Al margen de este hecho, no se constatan diferencias significativas entre unas zonas y otras. 
Tabla 10

Empleo creado por proyecto incentivado según tipo de zona

\begin{tabular}{|c|c|c|c|c|c|c|c|c|}
\hline & \multicolumn{5}{|c|}{ Zonas rurales } & \multirow{2}{*}{\multicolumn{2}{|c|}{$\begin{array}{c}\text { Zonas } \\
\text { Urbanas }\end{array}$}} \\
\hline & & A revitalizar & \multicolumn{2}{|c|}{ Intermedias } & \multicolumn{2}{|c|}{ Periurbanas } & & \\
\hline \multirow{3}{*}{ Descriptivo } & Promedio & 14,5 & \multicolumn{2}{|c|}{20,4} & \multicolumn{2}{|c|}{49,7} & \multicolumn{2}{|c|}{22,23} \\
\hline & Máximo & 240 & \multicolumn{2}{|c|}{495} & \multicolumn{2}{|c|}{1.065} & \multicolumn{2}{|c|}{450} \\
\hline & Mínimo & 0 & \multicolumn{2}{|c|}{0} & \multicolumn{2}{|c|}{0} & \multicolumn{2}{|c|}{0} \\
\hline \multicolumn{2}{|c|}{ Levene (significación) } & \multicolumn{7}{|c|}{$0,000^{*}$} \\
\hline \multirow{2}{*}{\multicolumn{2}{|c|}{ Anova (significación) }} & \multicolumn{7}{|c|}{$0,018^{\star}$} \\
\hline & & A revitalizar & Intern & edias & Periu & anas & Urbe & nas \\
\hline \multirow{3}{*}{$\begin{array}{c}\text { Prueba } \mathrm{T} \\
\text { (niveles de sig. } \\
\text { bilaterales) }\end{array}$} & A revitalizar & - & $0,023^{*}$ & 0,185 & $0,000^{*}$ & 0,208 & $0,001^{*}$ & $0,027^{*}$ \\
\hline & Intermedia & - & \multicolumn{2}{|c|}{ - } & $0,003^{*}$ & 0,317 & 0,614 & 0,709 \\
\hline & Periurbana & - & \multicolumn{2}{|c|}{-} & \multicolumn{2}{|c|}{-} & $0,001^{*}$ & 0,346 \\
\hline
\end{tabular}

(*) Nivel de significación: 0,05.

Fuente: Elaboración propia.

La tercera y última característica de los proyectos incentivados que debemos comparar es la inversión que está prevista realizar por cada empleo creado. Es decir, se pretende contrastar la siguiente hipótesis:

Ho: los proyectos incentivados a partir de 2008 realizan la misma inversión por empleo independientemente de la zona en que se localizan

A diferencia de las dos anteriores, la inversión que es preciso realizar por empleo (Tabla 11) es una característica de los proyectos incentivados que, por término medio, comparten las cuatro zonas consideradas $(\mathrm{p}=0,377)$. Ello no es óbice para que existan grandes diferencias dentro de cada zona observables cuando se comparan los valores máximos y los mínimos. La diferencia entre éstos supera los 5 millones de euros en las zonas rurales a revitalizar y los 36 millones en las urbanas.

Tabla 11

Inversión por empleo creado en los proyectos incentivado según tipo de zona

\begin{tabular}{|l|c|c|c|c|c|}
\hline \multicolumn{2}{|c|}{} & \multicolumn{3}{|c|}{ Zonas rurales } & Zonas \\
Urbanas
\end{tabular}

(*) Nivel de significación: 0,05.

Fuente: Elaboración propia. 
En definitiva, de los análisis efectuados podemos concluir que:

- no existen diferencias estadísticamente significativas en las características de los proyectos incentivados localizados en zonas rurales; $y$,

- la diferencia más notable se produce en el volumen de inversión media y en el empleo entre los proyectos incentivados en los municipios rurales a revitalizar y los municipios urbanos.

Cabe preguntarse, pues, si ante estas dos circunstancias han existido diferencias en las subvenciones otorgadas. Este hecho se examina en el epígrafe siguiente.

\section{CARACTERÍSTICAS DE LOS INCENTIVOS REGIONALES CONCEDIDOS TRAS LA APROBACIÓN DE LA LEY 45/2007}

Antes de realizar el análisis conviene recordar que la fortaleza de la Ley 50/1985 se basa en tratar de forma distinta a proyectos de inversión con características similares pero localizados en entornos geográficos dispares con el fin de revitalizar y potenciar las zonas más desfavorecidas. Con esta premisa, tratamos de averiguar si las subvenciones concedidas a partir de 2008 presentan diferencias significativas en función de la zona en que se localiza el proyecto. Para ello, examinamos tres elementos básicos de los incentivos concedidos, todos ellos en términos reales: la subvención por proyecto, el porcentaje de subvención concedido y la subvención otorgada por empleo creado.

La primera variable a analizar es la subvención concedida por proyecto y para ello sometemos a contraste la siguiente hipótesis:

Ho: la subvención es independiente de la zona en que se localiza el proyecto

En términos reales, la subvención concedida a los 627 proyectos incentivados supera los 561 millones de euros. El 53\% de esta cuantía (297,5 millones) ha servido para incentivar inversiones localizadas en zonas urbanas. Más de la mitad de la subvención localizada en el medio rural (139,2 millones) se ha concedido a empresas ubicadas en zonas intermedias, mientras que las zonas a revitalizar sólo han obtenido 87,4 millones, lo que supone el 15,6\% del volumen total de incentivos. Al margen de la distribución de la subvención total, el análisis de igualdad de medias recogido en la Tabla 12 permite, a partir del valor del nivel crítico ( $\mathrm{p}=0,097)$, aceptar la hipótesis nula y concluir que en las cuatro zonas analizadas, los proyectos tienen la misma subvención media. No obstante, a la luz de los resultados de la comparación bilateral entre zonas mediante la prueba $\mathrm{T}$ se debe matizar esta conclusión, apreciándose un cierto grado de disimilitud entre las zonas rurales a revitalizar y las urbanas $(p=0,010)$, mientras que la mayor semejanza se produce entre estas últimas y las periurbanas $(\mathrm{p}=0,804)$. Esta última circunstancia parece lógica dada la ya comentada semejanza en la inversión por proyecto (Tabla 9). Por otra parte, 
cuando se compara el primer trienio de la década con el último, la única variación significativa en esta característica se observa en las zonas intermedias $(\mathrm{p}=0,036)$.

Tabla 12

Subvención de los proyectos incentivados según tipo de zona

\begin{tabular}{|c|c|c|c|c|c|c|c|c|c|}
\hline & \multicolumn{6}{|c|}{ Zona rurales } & \multirow{2}{*}{\multicolumn{2}{|c|}{$\begin{array}{c}\text { Zonas } \\
\text { Urbanas }\end{array}$}} \\
\hline & & \multicolumn{2}{|c|}{ A revitalizar } & \multicolumn{2}{|c|}{ Intermedias } & \multicolumn{2}{|c|}{ Periurbanas } & & \\
\hline \multirow{3}{*}{ Descriptivo } & Promedio (€) & & .463 & \multicolumn{2}{|c|}{756.683} & \multicolumn{2}{|c|}{1.009 .916} & \multicolumn{2}{|c|}{1.139 .811} \\
\hline & Máximo $(€)$ & 6.5 & 3.505 & \multicolumn{2}{|c|}{12.679 .421} & \multicolumn{2}{|c|}{10.373 .886} & \multicolumn{2}{|c|}{41.735 .210} \\
\hline & Mínimo $(€)$ & & 056 & \multicolumn{2}{|c|}{30.781} & \multicolumn{2}{|c|}{66.703} & \multicolumn{2}{|c|}{32.062} \\
\hline \multicolumn{2}{|c|}{ Levene (significación) } & \multicolumn{8}{|c|}{$0,006^{*}$} \\
\hline \multirow{2}{*}{\multicolumn{2}{|c|}{ Anova (significación) }} & \multicolumn{8}{|c|}{0,097} \\
\hline & & A rel & italizar & Intern & edias & Periur & banas & Urba & inas \\
\hline \multirow{3}{*}{$\begin{array}{c}\text { Prueba } T \\
\text { (niveles de sig. } \\
\text { bilaterales) }\end{array}$} & A revitalizar & & - & 0,550 & 0,273 & $0,007^{*}$ & 0,209 & $0,040^{*}$ & $0,010^{*}$ \\
\hline & Intermedia & & - & \multicolumn{2}{|c|}{-} & 0,253 & 0,362 & 0,037 & 0,083 \\
\hline & Periurbana & & - & \multicolumn{2}{|c|}{-} & \multicolumn{2}{|c|}{ 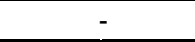 } & 0,679 & 0,804 \\
\hline \multicolumn{2}{|c|}{ Entre periodos } & $0,009 *$ & 0,071 & $0,001^{*}$ & $0,036^{*}$ & $0,011^{*}$ & 0,183 & 0,772 & 0,669 \\
\hline
\end{tabular}

$\left.{ }^{*}\right)$ Nivel de significación: 0,05.

Fuente: Elaboración propia.

La segunda variable a tener en cuenta es la relación entre la inversión prevista y la subvención concedida. Es decir, se pretende contrastar la hipótesis nula:

Ho: el porcentaje de subvención es independiente de la zona en que se localiza el proyecto

El porcentaje de subvención concedida no muestra, en principio, igualdad de medias en las cuatro zonas consideradas $(\mathrm{p}=0,002)$, ni homogeneidad de varianzas $(\mathrm{p}=0,012$ en la prueba de Levene) (Tabla 13). Los contrastes bilaterales revelan un comportamiento totalmente diferenciado entre la zona periurbana y el resto ( $\mathrm{p}=0,000$ en las tres pruebas). De hecho, el porcentaje medio de subvención en las zonas rurales a revitalizar, las zonas rurales intermedias y las zonas urbanas es muy similar $(12,4 \%, 12,1 \%$ y $11,9 \%$, respectivamente) por lo que resulta fácil entender que los niveles de significación bilaterales entre ellas alcancen altos valores. 
Tabla 13

Porcentaje de subvención de los proyectos incentivados según tipo de zona

\begin{tabular}{|c|c|c|c|c|c|c|c|c|c|}
\hline & \multicolumn{6}{|c|}{ Zonas rurales } & \multirow{2}{*}{\multicolumn{2}{|c|}{$\begin{array}{c}\text { Zonas } \\
\text { Urbanas }\end{array}$}} \\
\hline & & \multicolumn{2}{|c|}{ A revitalizar } & \multicolumn{2}{|c|}{ Intermedias } & \multicolumn{2}{|c|}{ Periurbanas } & & \\
\hline \multirow{3}{*}{ Descriptivo } & Promedio & & $4 \%$ & \multicolumn{2}{|c|}{$12,1 \%$} & \multicolumn{2}{|c|}{$8,8 \%$} & \multicolumn{2}{|c|}{$11,9 \%$} \\
\hline & Máximo & & $0 \%$ & \multicolumn{2}{|c|}{$30,0 \%$} & \multicolumn{2}{|c|}{$23,0 \%$} & \multicolumn{2}{|c|}{$29,0 \%$} \\
\hline & Mínimo & & & \multicolumn{2}{|c|}{$5,0 \%$} & \multicolumn{2}{|c|}{$5,0 \%$} & \multicolumn{2}{|c|}{$4,0 \%$} \\
\hline \multicolumn{2}{|c|}{ Levene (significación) } & \multicolumn{8}{|c|}{$0,012^{*}$} \\
\hline \multirow{2}{*}{\multicolumn{2}{|c|}{ Anova (significación) }} & \multicolumn{8}{|c|}{$0,002^{*}$} \\
\hline & & \multicolumn{2}{|c|}{ A revitalizar } & \multicolumn{2}{|c|}{ Intermedias } & \multicolumn{2}{|c|}{ Periurbanas } & \multicolumn{2}{|c|}{ Urbanas } \\
\hline \multirow{3}{*}{$\begin{array}{c}\text { Prueba T } \\
\text { (niveles de sig. } \\
\text { bilaterales) }\end{array}$} & A revitalizar & & & 0,817 & 0,604 & $0,002^{*}$ & $0,000^{*}$ & 0,432 & 0,413 \\
\hline & Intermedia & & & \multicolumn{2}{|c|}{ - } & $0,002^{*}$ & $0,000^{*}$ & 0,269 & 0,797 \\
\hline & Periurbana & & & \multicolumn{2}{|c|}{ - } & \multicolumn{2}{|c|}{ 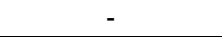 } & $0,006^{*}$ & $0,000^{*}$ \\
\hline \multicolumn{2}{|c|}{ Entre periodos } & $0,000^{*}$ & 0,541 & $0,000^{*}$ & 0,205 & 0,609 & 0,275 & $0,006^{*}$ & 0,183 \\
\hline
\end{tabular}

$\left.{ }^{\star}{ }^{\star}\right)$ Nivel de significación: 0,05.

Fuente: Elaboración propia.

Por otra parte, tal y como ya se ha señalado, uno de los pilares sobre los que se asienta la efectividad de los incentivos regionales es el de considerar prioritarios a determinados municipios dotándoles de la posibilidad de acceso al porcentaje de subvención máxima. Sin embargo, los datos no avalan que esta posibilidad se haya materializado, pues, salvo la zona periurbana el resto ha sido tratado de manera similar. Es más, el porcentaje de subvención más alto (30\%) se ha concedido a un proyecto localizado en una zona intermedia (Arjonilla, no prioritario según la Ley 45/2007 aunque sí según la Ley 50/1985) y las únicas cuatro subvenciones superiores al $25 \%$ se localizan en municipios urbanos (no prioritarios desde el punto de vista del desarrollo rural). No existen, tampoco, diferencias significativas entre ambos periodos cuando se considera cada zona de forma particularizada.

La tercera y última variable a considerar es la relación entre el empleo creado y la subvención concedida. Es decir, se pretende contrastar la hipótesis nula:

Ho: la subvención por empleo es independiente de la zona en que se localiza el proyecto

De acuerdo con los datos de la Tabla 14, en general, no se detectan diferencias significativas en esta característica $(\mathrm{p}=0,361)$, sin embargo, una comparación bilateral entre las cuatro zonas hace aflorar importantes diferencias en el medio rural, especialmente, entre las zonas a revitalizar y las periurbanas $(\mathrm{p}=0,011)$ y entre éstas y las zonas intermedias $(\mathrm{p}=0,003)$. De hecho, la subvención por empleo de los proyectos periurbanos se asemeja más al de los urbanos que al del resto de áreas rurales. Dentro de cada zona no existe una 
homogeneidad en los proyectos, observándose importantes diferencias en el interior de cada zona. Así, por ejemplo, en el entorno urbano encontramos un proyecto en el que la subvención por empleo supera los 3,6 millones de euros junto a otro que no alcanza los 500 euros. Paralelamente, en la zona rural a revitalizar la subvención mínima concedida por empleo es de 2.021 euros y la máxima de 932.000 euros. En todo caso, debemos recordar, por una parte, que la Ley de Incentivos Regionales no subvenciona los empleos creados sino determinados tipos de inversiones $\mathrm{y}$, por otra, que muchas de estas inversiones no implican, necesariamente, la creación de nuevos puestos de trabajo. En la subvención por empleo no todas las zonas se comportan de manera similar en los dos periodos considerados. Esta diferencia se produce en las zonas urbanas $(\mathrm{p}=0,043)$ y especialmente en las intermedias $(\mathrm{p}=0,001)$.

Tabla 14

Subvención por empleo de los proyectos incentivados según tipo de zona

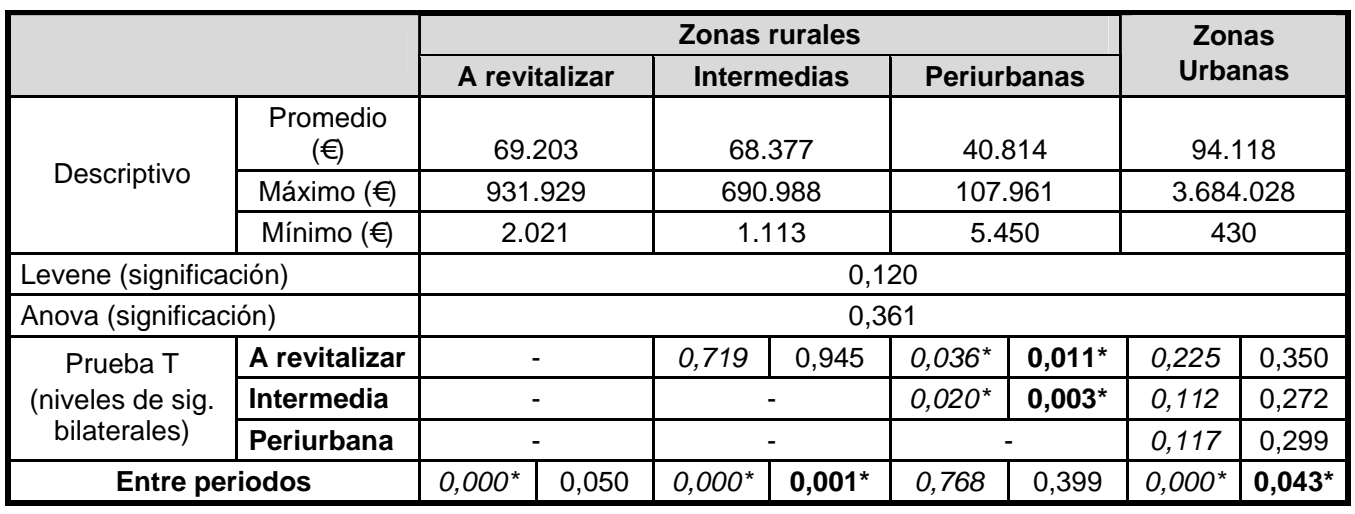

(*) Nivel de significación: 0,05.

Fuente: Elaboración propia.

En definitiva, salvo diferencias en la subvención media entre los proyectos incentivados en los municipios rurales a revitalizar y los municipios urbanos, y en el porcentaje de subvención concedido entre las zonas periurbanas y el resto, podemos asumir una similitud en las características de la subvención concedida a los proyectos de inversión.

\section{CONCLUSIONES}

La lectura de las siguientes conclusiones debe estar precedida por dos consideraciones. En primer lugar, que la utilización de los incentivos regionales con el objetivo de fomentar la actividad económica en el medio rural no es efectiva hasta la entrada en vigor, a mediados de 2010, del primer Programa de 
Desarrollo Rural Sostenible y, en segundo lugar, que el objetivo de este trabajo no ha sido el contrastar su efectividad, sino el de comprobar si durante el escaso periodo de tiempo transcurrido desde la aprobación de la Ley 45/2007 hasta la entrada en vigor del citado Programa se observan indicios que permitan aventurar algún cambio de tendencia en la forma y en la dirección en que se han aplicado los incentivos regionales.

Desde el punto de vista operativo se constata que a pesar de las evidentes diferencias en circunstancias y problemas que presentan las zonas del país, todos los Reales Decretos de delimitación de las trece zonas de promoción económica, coinciden en objetivos, sectores y elementos incentivables. La Ley para el Desarrollo Sostenible del Medio Rural es de aplicación en todo el territorio nacional, pero los incentivos regionales como instrumento al servicio de ésta sólo pueden aplicarse en una parte del territorio; es decir, el Sistema Nacional de Incentivos Regionales no puede emplearse como instrumento para fomentar la actividad económica en todo el territorio rural a revitalizar. Desde su nacimiento y como consecuencia del origen de sus fondos (Fondo Europeo de Desarrollo Regional), Baleares, Cataluña, Madrid, Navarra y La Rioja quedaron excluidas del ámbito de aplicación de la Ley 50/1985, a las que posteriormente se unió el País Vasco. En concreto, el Programa de Desarrollo Rural Sostenible considera 325 municipios de primer nivel (prioritarios) que se localizan en Comunidades no incentivables: Cataluña (140), Navarra (90) y La Rioja (95). En otros términos, la Ley 45/2007 se dota de un instrumento no aplicable en el 8,4\% de los municipios, el 5,3\% de la superficie, y el 3,9\% de la población de España.

Además de las cuestiones operativas, los análisis realizados en las páginas anteriores permiten extraer las siguientes conclusiones:

- En general, las características de los proyectos incentivados tras la aprobación de la Ley para el desarrollo sostenible del medio rural no difieren sustancialmente de los subvencionados durante el primer trienio de vigencia del Programa Operativo de Mejora de la Competitividad y Desarrollo del Tejido Productivo.

- En los tres tipos de zonas rurales (a revitalizar, intermedias y periurbanas) los proyectos comparten características básicas respecto a inversión y empleo. Además, salvo en la zona rural intermedia, estas características son similares a las de los proyectos incentivados entre 2000 y 2002.

- En particular, la diferencia más notable se produce en el tamaño de los proyectos, tanto si este se mide por la inversión como por el empleo, entre los proyectos localizados en los municipios rurales a revitalizar y los municipios urbanos En estos últimos, la dimensión media de los proyectos duplica a los incentivados en los municipios rurales a revitalizar. 
- No se aprecian diferencias sustanciales en las características de los incentivos concedidos en función de su localización ni entre zonas rurales, ni entre éstas y las áreas urbanas. De manera particular, cabe mencionar alguna discrepancia entre los municipios rurales a revitalizar y los urbanos en relación a la subvención media, y entre las zonas periurbanas y el resto de zonas respecto al porcentaje de subvención concedida. En todo caso, estas diferencias deben ser atribuidas a la diferencia en el tamaño de los proyectos antes mencionada y no a una decisión efectiva de beneficiar a las inversiones de una zona determinada.

- Los municipios rurales más desfavorecidos, a pesar de ser prioritarios, no se han visto discriminados positivamente por el porcentaje de subvención concedida. Es más, ninguno de los siete mayores porcentajes de subvención se dirige a una zona rural a revitalizar.

En definitiva, durante los tres años transcurridos entre la aprobación de la Ley para el desarrollo sostenible del medio rural y el Programa de Desarrollo Rural Sostenible no se aprecia un cambio significativo de tendencia que permita aventurar que los incentivos regionales comiencen a dirigirse mayoritaria y preferentemente hacia las zonas rurales más desfavorecidas (prioritarias). El propio Programa proporciona la explicación a esta circunstancia cuando señala la imposibilidad de determinar a priori la parte del presupuesto que se aplicará en las zonas prioritarias pues ello dependerá de los proyectos presentados y sus características. Este hecho cuestiona la utilización de los incentivos regionales como instrumento, por cuanto, no serán efectivos si no se solicitan y sólo se solicitarán allí donde ya exista una "masa critica" de empresas y población, aspecto éste que no suele darse en el medio rural por lo que resulta difícil que de estas zonas partan las solicitudes necesarias previas a la recepción de los incentivos.

\section{REFERENCIAS BIBLIOGRÁFICAS}

ALEIXANDRE MENDIZÁBAL, G. Y MOYANO PESQUERA, P.B. (2009): La creación de empresas en Castilla y León: factores condicionantes a escala provincial. Junta de Castilla y León. Mimeo.

ALEIXANDRE MENDIZÁBAL, G. Y MOYANO PESQUERA, P.B. (2010): Factores condicionantes de la creación de empresas para las provincias españolas. Internacional Meeting on Regional Science. Badajoz, 17-19 de noviembre.

ARMINGTON, C. Y ACS, Z.J. (2002): "The determinants of regional variation in new firm formation". Regional Studies, vol. 36.1, pp. 33-45. 
AUDRETSCH, D.B. Y FRISTCH, M. (1994): "The geography of firm births in Germany". Regional Studies, vol. 28.4, pp. 359-365.

AUDRETSCH, D.B. Y FRITSCH, M. (1999): "The industry component of regional new firm formation processes". Review of Industrial Organization, vol. 15.3, pp. 239-252.

BADE, F.J. Y NERLINGER, E.A. (2000): "The spatial distribution of new technology-based firms: Empirical results for West-Germany". Papers in Regional Science, 79, pp. 155-176.

BOIX DOMĖNECH, R. (2007): Concepto y delimitación de áreas metropolitanas: una aplicación a las áreas metropolitanas de España. Seminario sobre "Las grandes áreas metropolitanas españolas en una perspectiva comparada". Centro de Estudios Andaluces. Consejería de la Presidencia. Junta de Andalucía. Sevilla, 11 de Abril.

BRYANT, C.R. (1973): "Urbanisation et structures agricoles de la région parisienne entre 1955 et 1966. Essay de méthode". Etudes rurales, $n^{\circ}$ 49/50, L'urbanisation des Campagnespp. pp. 205-244.

BRYANT, C.R. (1974): "An approach to the problem of urbanisation and structural change in agriculture: a case study from de Paris Region, 1955 to 1968". Geografiska Annaler 56B, pp. 1-27.

CLOKE, P. (1977): "An Index of Rurality for England and Wales". Regional Studies, 11. pp. 37-46

CLOKE, P. Y EDWARDS, G. (1986): "Rurality in England and Wales 1981: A Replication of the 1971 Index". Regional Studies, vol. 20.4. pp. 289-306.

COMISIÓN EUROPEA (1979): Comunicación de la Comisión sobre los regímenes de ayudas de finalidad regional de 21 de diciembre de 1978. (DO C 031 de 3 de febrero de 1979).

COMISIÓN EUROPEA (1995): Carta de la Comisión al Gobierno español de 7 de septiembre de 1995 de aprobación del mapa de ayudas con finalidad regional para el periodo 1995-1999. (SG(1995) D/11308).

COMISIÓN EUROPEA (1998): Directrices sobre las ayudas de Estado de finalidad regional. (DOC 74/06 de 10 de marzo de 1998).

COMISIÓN EUROPEA (2000): Carta de la Comisión al Gobierno español de 17 de mayo de 2000 de aprobación del mapa de ayudas con finalidad regional para el periodo 2000-2006. (SG(2000) D/103727)

COMISIÓN EUROPEA (2006): Directrices sobre las ayudas de Estado de finalidad regional para el período 2007-2013. (DOC 54/08 de 4 de marzo de 2006).

COMISIÓN EUROPEA (2007): Directrices sobre las ayudas de Estado de finalidad regional para el período 2007-2013. Mapa de Ayudas Regionales. España. (DOC 35/04 de 17 de febrero de 2007).

DAVIDSSON, P.; LINDMARK, L. Y OLOFSSON, C. (1994): "New firm formation and regional development in Swenden". Regional Studies, vol. 28.4, pp. 395-410. 
DEFRA (2004): Rural Strategy 2004. Disponible en: http://www.defra.gov.uk/rural/documents/policy/strategy/rural_strategy_2004 .pdf. Último acceso: 1 de enero de 2011.

EUROSTAT (1999): The densely populated areas in the European Union Towards a delimitation and characterisation of urban areas. Statistics in focus. General statistics. Theme $1-2 / 1999$.

EUROSTAT (2004): Urban Audit. Methodological Handbook. European Commission. Office for Official Publications of the European Communities, Luxembourg.

FARIÑA GÓMEZ, B.; GORDO GÓMEZ, P.; RÍOS RODICIO, A. Y RODRÍGUEZ PRADO, B. (2004): Identificación y tipificación de los espacios rurales en la comunidad autónoma de Castilla y León. Una aproximación para el análisis de las dinámicas espaciales. V Congreso de Economía Agraria. Santiago de Compostela. Disponible en: http://www.emp.uva.es/ pablog/ espacio_rural_cl.pdf. Último acceso: 2 de enero de 2010.

FERIA TORIBIO, J.M. (2004): "Problemas de definición de las áreas metropolitanas en España". Boletín de la Asociación de Geofráfos Españoles, 38, pp. 85-99

FRITSCH, M. Y MUELLER, P. (2007): "The persistence of regional new business formation-activity over time - assessing the potential of policy promotion programs". Journal of Evolutionary Economics, vol. 17.3, pp. 299315.

GARAFOLI, G. (1994): "New firm formation and regional development: The Italian case". Regional Studies, vol. 28.4. pp. 381-393.

GEORGELLIS, Y. Y WALL, H.J. (2000): "What makes a region entrepreneurial? Evidence from Britain". The Annals of Regional Science, 34, pp. 385-403.

Guesnier, B. (1994): "Regional variations in new firm formation in France". Regional Studies, vol. 28.4, pp. 347-358.

HARRINGTON, V. Y O'DONOGHUSE, D. (1998): "Rurality in England and Wales 1981: A Replication an Extension of the 1981 Rurality Index". Sociologia Ruralis, vol. 38.2, pp. 178-203.

HART, M. Y GUDGIN, G. (1994): "Spatial variations in new firm formation in the Republic of Ireland, 1980-1990". Regional Studies, vol. 28.4, pp. 367-380.

KANGASHARJU, A. (2000): "Regional variations in firm formation: Panel and cross-section data evidence form Finland". Papers in Regional Science, vol. 79.4, pp. 355-373.

KEEBLE, D. Y WALKER, S. (1994): "New firms, samll firms and dead firms: spatial patterns and determinants in the United Kingdom". Regional Studies, vol. 28.4, pp. 411-427.

MALUQUER I AMOROS, S. Y POVEDA MARTÍNEZ, C. (2000): "La nueva zona de ayudas de Estado con finalidad regional en España". Boletín Económico de ICE, $\mathrm{n}^{\circ} 2661$, pp.43-52. 
MINISTERIO DE VIVIENDA (2006): Atlas Estadísticos de las Áreas Urbanas de España 2006. Secretaría General Técnica. Centro de Publicaciones. Madrid MINISTERIO DE VIVIENDA (2009): Atlas Estadísticos de las Áreas Urbanas de España 2009. Secretaría General Técnica. Centro de Publicaciones. Madrid. Disponible en http://siu.vivienda.es/portal/index.php?option=com_content\&view= category\&layout=blog\&id=19\&ltemid=73\&lang=es. Consultado el 31 de diciembre de 2010.

MINISTERIO DE MEDIO AMBIENTE Y MEDIO RURAL Y MARINO (2010): Plan Estratégico Nacional de Desarrollo Rural.

MINISTERIO DE MEDIO AMBIENTE Y MEDIO RURAL Y MARINO (2010): Programa de Desarrollo Rural Sostenible para el periodo 2010-2014. Real Decreto 752/2010 de 4 de junio. BOE no 142 de 11 de junio de 2010.

MOYANO PESQUERA, P.B. Y OGANDO CANABAL, O. (2003): "La instrumentación de la Política Regional española en la última década: el papel de los incentivos económicos a la inversión y al empleo". Revista de Estudios Regionales, $\mathrm{n}^{\circ}$ 68. $2^{\mathrm{a}}$ época, pp. 15-38.

MOYANO PESQUERA, P.B.; ALEIXANDRE MENDIZABAL, G. Y OGANDO CANABAL, O. (2006): "Análisis de los resultados de las medidas de fomento económico regional a nivel municipal". Investigaciones Regionales, 9, pp. 115-136.

MOYANO PESQUERA, P.B. Y ALEIXANDRE MENDIZABAL, G. (2009): La aplicación del Programa de Mejora de la Competitividad y Desarrollo del Tejido Productivo en Castilla y León: análisis de los efectos sectoriales y territoriales. Consejería de Economía y Hacienda de la Junta de Castilla y León. Mimeo

NYSTRÖM, K. (2007): "An industry disaggregated analysis of the determinants of regional entry and exit". Annals of Regional Science, vol. 41.4. pp. 877896.

OCDE (2005): Regions at a Glance 2005. OECD Publications, Paris.

OCDE (2009): Regions at a Glance 2009. OECD Publications, Paris.

PANIAGUA, A. (2004): "La geografía rural, entre el peso de la regulación y las orientaciones constructivistas". Document's d'Analisi Geográfica, 43. pp. 123-134.

PANIAGUA, A. (2010) Repensar el espacio (rural): entre la individualización y el Campo global. VIII Congreso Latinoamericano de Sociología Rural, Porto de Galinhas, 2010.

PANIAGUA, A. Y HOGGART, K. (2002): "Lo rural, ¿Hechos, discursos o representaciones? Una perspectiva geográfica de un debate clásico". Información Comercial Española, $n^{\circ}$ 803, pp. 61-71.

RODRÍGUEZ A. Y SABORÍO, M. (2008): "Algunas consideraciones conceptuales y metodológicas sobre la definición y medición de lo rural". En Rodríguez A. y Saborío, M. (Eds.): Lo rural es diverso. Evidencia para el 
caso de Costa Rica. Instituto Interamericano de Cooperación para la Agricultura (IICA). San José (Costa Rica). Disponible en www.iica.int.

SEREMI (2003): Actualización metodología de asignación comunal y provincial de la inversión: Seremi de planificación metropolitana. Disponible en: http://www.serplacrm.

cl/publicaciones/metodologias/metodologia_asignacion_comunal_dic2003.p df. Último acceso: 3 de enero de 2011.

SERRANO MARTÍNEZ, J.M. (2006): "Aglomeraciones y áreas urbanas en España, dimensión y tendencias: breves precisiones". Lurralde: Investigación y espacio, 29, pp. 115-142.

TIÓ SARALEGUI, C. (2005): Situación actual y perspectivas de desarrllo del mundo rural en España. Documento de trabajo 74(2005). Fundación Alternativas. Madrid.

ZAMUDIO SÁNCHEZ, F.J.; Corona Ambriz. A. y López Becerril, I.D. (2008): "Un índice de ruralidad para México" Espiral, Estudios sobre Estado y Sociedad Vol. XIV No. 42. pp. 179-214. 